C.R. Harington - Quaternary cave faunas of Canada: a review of the vertebrate remains. Journal of Cave and Karst Studies, v. 73, no. 3, p. 162-180. DOI: $10.4311 /$ jcks2009pa128

\title{
QUATERNARY CAVE FAUNAS OF CANADA: A REVIEW OF THE VERTEBRATE REMAINS
}

\author{
C.R. HARINGTON \\ Canadian Museum of Nature (Paleobiology),Ottawa K1P6P4 Canada, dharington@mus-nature.ca
}

\begin{abstract}
Highlights of ice-age vertebrate faunas from Canadian caves are presented in geographic order (east to west). They include four each from Quebec and Ontario; three from Alberta; one from Yukon; and ten from British Columbia. Localities, vertebrate species represented, radiocarbon ages, and paleoenvironmental evidence are mentioned where available, as well as pertinent references. Of these caves, perhaps Bluefish Caves, Yukon, are most significant, because they contain evidence for the earliest people in North America. Tables provide lists of species and radiocarbon ages from each site.
\end{abstract}

\section{INTRODUCTION}

Although some cave faunas from the United States and Mexico are dealt with in the book Ice Age Cave Faunas of North America (Schubert et al., 2003), no Canadian cave faunas are mentioned. To help broaden that perspective, highlights of twenty-two Quaternary vertebrate faunas from Canadian caves (Fig. 1) are summarized here, progressing geographically from east to west. This paper developed from a review of the 2003 book (Harington, 2006).

It is important to study vertebrate faunal remains from Canadian caves for several reasons. Specimens often preserve well in a stratified sedimentary context, allowing a better understanding of the environments occupied by various animals represented. If such strata are sufficiently deep and discrete (bioturbation and cryoturbation can cause problems), they help us understand how climate, environment, and fauna have changed through time, such as across the Pleistocene/Holocene boundary finely recorded at Bluefish and Charlie Lake caves. Furthermore, Canada has been subject to particularly heavy, multiple glaciations in the Quaternary that have greatly disturbed fossil sites on open terrain, with fragmenting of fossil bones. So it is valuable to have pockets such as caves, fissures, and rock shelters in upland areas from coast to coast that can preserve long faunal and environmental records. It is especially valuable to have microfaunal remains, including fragile bones of ecologically-sensistive fish, amphibians, reptiles, and rodents, often well-preserved in quiet cave surroundings that are available for study.

Apart from presenting highlights of the cave faunas and their importance to Canadian Quaternary studies, generally, the purpose of this paper is to list the taxa in each vertebrate fauna and provide radiocarbon ages for them (Tables 1 and 2), as well as illustrating some of the caves.

\section{LOCALITIES}

QuEBEC

Caves near Saint-Elzéar and La Rédemption in the Gaspésie region, as well as Mine and Laflèche caves in
Gatineau, have yielded fascinating glimpses of Québec's Quaternary faunas (for summaries see Beaupré and Caron, 1986; and Harington, 2003a; Fig. 1a).

Saint-Elzéar Cave $\left(48^{\circ} 14^{\prime} 20^{\prime \prime} \mathrm{N}, 65^{\circ} 21^{\prime} 30^{\prime \prime} \mathrm{W}\right)$, situated on a plateau north of Baie des Chaleurs, has produced remains of three species of amphibians, one species of reptile, four species of birds, and thirty-four species of mammals. About $80 \%$ of nearly 4,700 fossils are fragments of small mammals. Several species no longer live in the region, and a period of colder climate seems to be indicated by the presence of some of them, such as the arctic shrew Sorex arcticus, the arctic hare Lepus arcticus, the western heather vole Phenacomys intermedius, the Ungava collared lemming Dicrostonyx hudsonius, and the least weasel Mustela nivalis. Due to soil chemistry, the bones seem unsuitable for radiocarbon dating (LaSalle, 1984; LaSalle and Guilday, 1980). However, a few of the cold-adapted species might be sampled for AMS radiocarbon dating, because they are likely to be relatively old.

Preliminary collections of faunal remains from sediments in caves near La Rédemption (Trou Otis and Spéos de la Fée; $48^{\circ} 27^{\prime} \mathrm{N}, 67^{\circ} 50^{\prime} \mathrm{W}$ ) yielded seven mammal species: Keen's bat Myotis keenii, the Ungava lemming, the North American porcupine Erethizon dorsatum, the red fox Vulpes vulpes, the brown bear Ursus arctos, the caribou Rangifer tarandus, and the moose Alces alces). The lemming and brown bear are of great interest because of their rarity in the faunal record of eastern North America. I postulated that they occupied Gaspésie when patches of tundra-like habitat existed there, perhaps in early postglacial time (Harington, 1980). A radiocarbon date on the bear specimen did not yield conclusive information.

Located some $20 \mathrm{~km}$ northeast of Ottawa, Mine Cave $\left(45^{\circ} 28^{\prime} 18^{\prime \prime} \mathrm{N}, 75^{\circ} 51^{\prime} 01^{\prime \prime} \mathrm{W}\right.$; Fig. 2) is a natural trap that has accumulated many bones and teeth that are useful for paleoecological reconstruction. It lies on the Eardley Escarpment within the limits of Gatineau Park. The upper $100 \mathrm{~cm}$ of the infill, containing relatively recent fauna dating back to about 5,000 B.P. (radiocarbon years before present, taken as 1950), is characterized by the American black bear Ursus americanus, the white- 

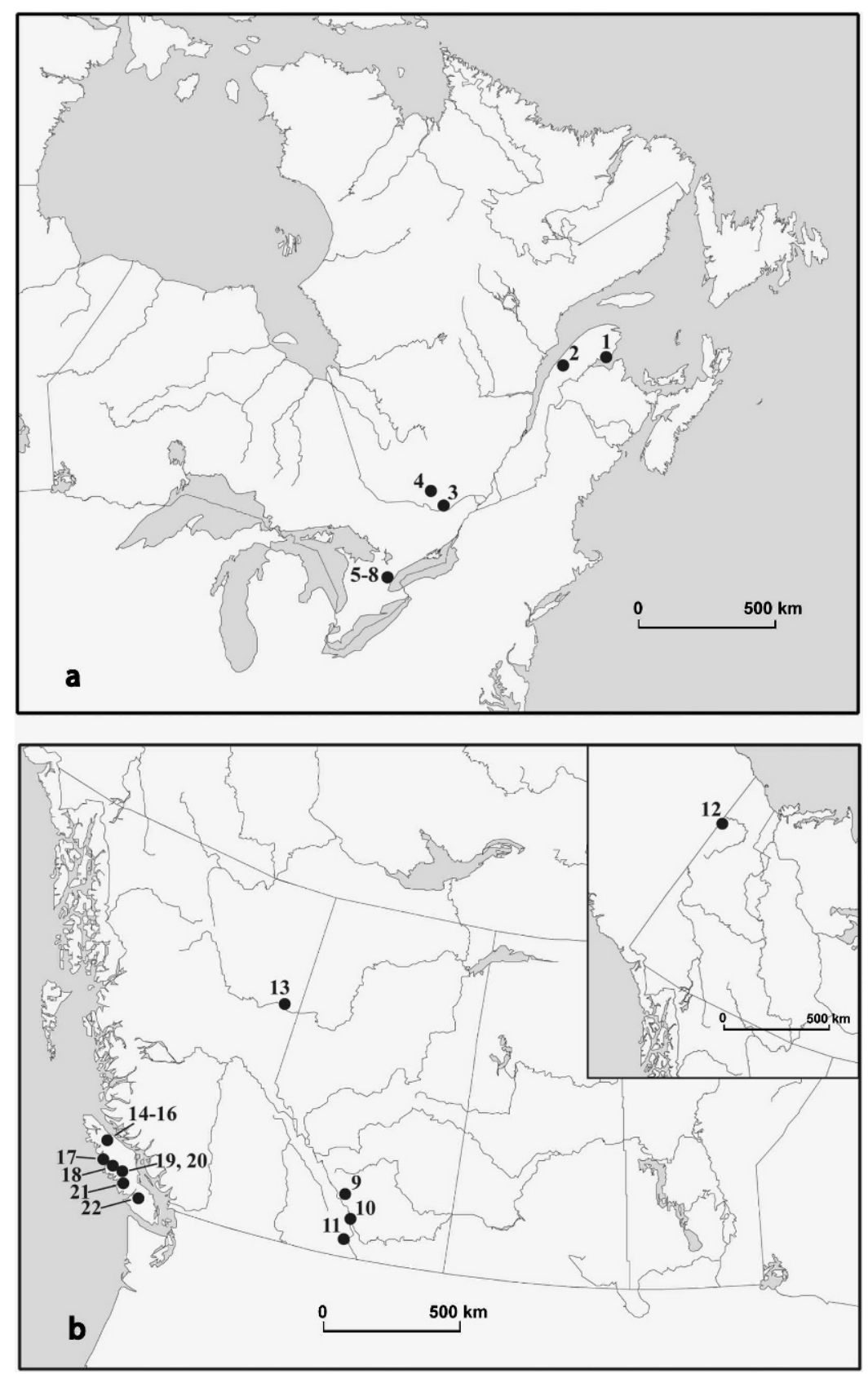

Figure 1. Maps showing sites of most important Quaternary vertebrate faunas in Canadian caves. (a) Eastern Canada. Quebec: 1 Saint-Elzéar Cave, 2 La Rédemption caves (Trou Otis, Spéos-de-la-Fée), 3 Mine Cave, 4 Laflèche Cave. Ontario: 5 Elba Cave, 6 Kelso Cave, 7 Mt. Nemo Cave, 8 Dickson Cave. (b) Western Canada. Alberta: 9 Rats Nest Cave, 10 January Cave, 11 Eagle Cave. Yukon (inset): 12 Bluefish Caves. British Columbia: 13 Charlie Lake Cave, 14 Resonance Cave, 15 Pellucidar I Cave, 16 Pellucidar II Cave, 17 Port Eliza Cave, 18 Windy Link Pot Cave, 19 Mariner Mountain Cave, 20 Golden Hinde Cave, 21 Clayoquot Plateau Cave, 22 Limestone Mountain Cave.

tailed deer Odocoileus virginianus, the raccoon Procyon lotor, the deer mouse Peromyscus sp., and the big brown bat Eptesicus fuscus (Carrier, 1989). The bottom $70 \mathrm{~cm}$ of infill dates between 8,230 \pm 80 and 5,020 \pm 70 B.P., and it contains remains of amphibians, reptiles (snakes), and twenty-three species of mammals, but no black bear or deer. Two species, the woodland vole Microtus pinetorum and the Ungava lemming, no longer occupy the area. The former is found mainly in the eastern United States, whereas the latter occurs in northern Québec and Labrador, suggesting Holocene range shifts to the south and north, respectively. Most small mammals from the lower infill consist of boreal species (Lauriol et al., 2003).

Journal of Cave and Karst Studies, December 2011 163 
Laflèche Cave $\left(45^{\circ} 39^{\prime} 00^{\prime \prime} \mathrm{N}, 75^{\circ} 46^{\prime} 48^{\prime \prime} \mathrm{W}\right)$ is about $27 \mathrm{~km}$ north of Gatineau (formerly Hull). Upper and lower levels are joined by an $18 \mathrm{~m}$ vertical shaft. Sandy matrix up to $80 \mathrm{~cm}$ deep near the lower (man-made) entrance has yielded four birds and twenty-one species of mammals (Harington, 2003). Several arctic-adapted species are represented in this fauna, such as the snowy owl Nyctea scandiaca, the arctic hare, the arctic fox Alopex lagopus, and the Ungava lemming, suggesting the presence of tundra-like conditions following the retreat of Laurentide ice from the region in late glacial time. Indeed, an arctic fox mandible yielded a date of $10,800 \pm 90$ B.P.; an unidentified bone yielded a comparable date of 9,310 \pm 80 B.P. It is worth noting that such species retreated northward with the melting Laurentide Ice Sheet, and at present, they survive in northernmost Quebec and Labrador. Perhaps the archaeological and historical evidence for brown bears (Ursus arctos) in Labrador and northern Quebec fits this hypothesis, too (Pigott, 1999).

\section{ONTARIO}

Four caves worth mentioning in Ontario (Fig. 1a) are Elba, Kelso, Mt. Nemo, and Dickson. Faunal remains for each were summarized by Churcher and Karrow (2008, Table 1). They are situated on the Niagara Escarpment. Elba Cave $\left(44^{\circ} 01^{\prime} \mathrm{N}, 80^{\circ} 10^{\prime} \mathrm{W}\right)$ is a fissure deposit in Mono Cliffs Provincial Park in south-central Ontario. The entrance is vertical. Howard Savage (1994) and crew collected 625 bones of twenty mammalian species from various ledges. Eighteen of the twenty taxa are typical of the present-day fauna of the region. Only two, the pika Ochotona sp. and the American marten Martes americana (locally exterminated in the early 1900s), are not part of the current local community. In such crevice or fissure faunas, the crevices open and close because of karstic solution, rock movement, or infilling by calcium carbonate dripstone (Karrow, 2005), allowing animal remains to be deposited there at more than one time. Thus a single large (extinct giant?) pika femur from a ledge $25 \mathrm{~m}$ below the surface in Elba Cave was AMS dated at 8,670 \pm 220 B.P., and an American marten skull from that cave yielded a date of $510 \pm 60$ B.P.

Remains of vertebrates were found in a travertinecemented dolomite breccia from a demolished cave (Kelso Cave; $43^{\circ} 30^{\prime} \mathrm{N}, 70^{\circ} 55^{\prime} \mathrm{W}$ ) in the Niagara Escarpment west of Milton, Ontario. They represent the American toad Bufo americanus, the spruce grouse Canachites canadensis or the ruffed grouse Bonasa umbellus, the snowshoe hare Lepus americanus, the eastern cottontail Sylvilagus floridanus, the giant pika, the woodland deer mouse Peromyscus maniculatus, the muskrat Ondatra zibethicus, the little brown bat Myotis lucifugus, and the striped skunk Mephitis mephitis. Pollen remains from the matrix with the extinct giant pika Ochotona whartoni bone suggest an age of about 10,000 years. Therefore, Mead and Grady (1996) suggest that the giant pika lived, possibly relictually, in southern Ontario into the Early Holocene.
Mt. Nemo Cave was the first of these crevice caves reported (Bateman, 1961). Its fauna consists of two species of bats, the North American beaver Castor canadensis, and the white-tailed deer. Because of the cave's elevation, the last two species may have been introduced by a predator that denned in it (Churcher and Karrow, 2008).

The fauna of Dickson Cave, situated in the Niagara Escarpment north of Mt. Nemo, consists of the painted turtle Chrysemys picta, the northern short-tailed shrew Blarina brevicauda, the smoky shrew Sorex fumeus, the big brown bat, the long-eared bat, the southern red-backed vole Myodes (Clethrionomys) gapperi, the meadow vole Microtus pennsylvanicus, the red squirrel Tamiasciurus hudsonicus, the woodland deer mouse, the muskrat, and the wapiti Cervus elaphus. The fauna is considered to be older than AD 1750 based on the presence of wapiti (Churcher and Fenton, 1968; Churcher and Karrow, 2008).

\section{Alberta}

In Alberta (Fig. 1b), the faunal remains from January and Eagle caves are best known. January Cave $\left(50^{\circ} 11^{\prime} \mathrm{N}\right.$, $114^{\circ} 31^{\prime} \mathrm{W}$ ), at an elevation of $2,040 \mathrm{~m}$, is located on Plateau Mountain in the Front Range of the Rocky Mountains, and is about $100 \mathrm{~km}$ southeast of Calgary (Fig. 3). The six most common vertebrate remains represented in the cave are the pika, the hoary marmot Marmota caligata, the Columbian ground squirrel Spermophilus columbianus, the bushy-tailed wood rat Neotoma cinerea, the long-tailed vole Microtus longicaudus, the deer mouse, and the arctic grayling Thymallus arcticus. A shrew Sorex sp., a hare Lepus sp., the northern pocket gopher Thomomys talpoides, several small carnivores, and the bighorn sheep Ovis canadensis are also represented. Roughly $90 \%$ of all identified fossils are rodents; probably most were derived from raptor pellets and carnivore droppings. Four exotic species (brown lemming Lemmus sibiricus, collared lemming Dicrostonyx torquatus, Niobrara prairie dog Cynomys cf. C. niobrarius, and blackfooted ferret Mustela nigripes) were identified. The lemmings, presently occupying northern Canada, and the ferret and prairie dog, characteristic of a more southern range in the United States plains, were found together, and radiocarbon dates on bone of mixed species extend from about 33,000 to 23,000 B.P., so probably the fauna represents a cool, dry mid-Wisconsin interstadial (Burns, 1991, 2002).

Eagle Cave $\left(49^{\circ} 38^{\prime} \mathrm{N}, 114^{\circ} 38^{\prime} \mathrm{W}\right)$ is situated about $10 \mathrm{~km}$ west of Coleman, Alberta, at an elevation of $1,377 \mathrm{~m}$ in Crowsnest Pass. Like January Cave, the sediments were deposited during the mid-Wisconsin (more than 33,000 to 23,000 B.P.). The northern pocket gopher Thomomys talpoides and the water vole Microtus richardsoni are perhaps the most interesting species in the fauna. The water vole fossil is the oldest known of the species, and its presence in southwestern Alberta suggests equable climatic conditions during the mid-Wisconsin. The pocket gopher is 
Table 1. Quaternary cave faunas of Canada: vertebrate taxa from selected localities.

Localities $^{\mathrm{a}}$

Cave Faunas

$\begin{array}{llllllllllllllllllllll}1 & 2 & 3 & 4 & 5 & 6 & 7 & 8 & 9 & 10 & 11 & 12 & 13 & 14 & 15 & 16 & 17 & 18 & 19 & 20 & 21 & 22\end{array}$

Fishes

Arctic grayling (Thymallus arcticus)

Longnose sucker (Catostomus catostomus)

Inconnu (Stenodus leucichthys)

Northern pike (Esox lucius)

Salmon (Onchorhynchus sp.)

Cuthroat trout (Onchorhynchus clarkii)

Greenling (Hexagrammidae)

Alaska pollock (Theragra chalcogramma)

Flatfish (Pleuronectiformes)

Irish lord (Hemilepidotus sp.)

Sculpin (Cottidae)

Pacific tomcod (Microgadus proximus)

Three-spined stickleback

(Gasterostens aculeatus)

Amphibians

Blue-spotted salamander (Ambystoma laterale)

American toad (Bufo americanus)

Western toad (Bufo boreas)

Toad (Bufo sp.)

Woodfrog (Rana palustris)

Bullfrog (Rana catesbiana)

Frog (Rana sp.)

Frogs (Anura)

Reptiles

Snakes (Serpentes)

Painted turtle (Chrysemys picta)

Birds

Red-throated loon (Gavia stellata)

Western grebe (Aechmophorus occidentalis)

Red-necked grebe (Podiceps grisegena)

Horned grebe (Podiceps auritus)

Eared grebe (Podiceps negricollis)

Grebe (Podicepedidae)

Double-crested cormorant

(Phalacrocorax auritus)

Cormorant (Phalacrocorax sp.)

Swan (Cygnini)

Canada goose (Branta canadensis)

Snow goose (Chen caerulescens)

Geese (Anserini)

Mallard (Anas platyrhynchos)

$\begin{array}{lllllllllllllllllllllll}\cdots & \cdots & \cdots & \cdots & \cdots & \cdots & \cdots & \cdots & \mathrm{x} & \cdots & \mathrm{x} & \mathrm{x} & \mathrm{x} & \cdots & \cdots & \cdots & \mathrm{x} & \cdots & \mathrm{x} & \cdots & \cdots & \cdots\end{array}$

$\begin{array}{llllllllllllllllllllllll}\cdots & \cdots & \cdots & \cdots & \cdots & \cdots & \cdots & \cdots & \cdots & \cdots & \cdots & \mathrm{x} & \mathrm{ef} & \cdots & \cdots & \cdots & \cdots & \cdots & \cdots & \cdots & \cdots & \cdots\end{array}$

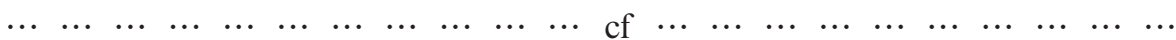

$\begin{array}{llllllllllllllllllllll}\ldots & \ldots & \ldots & \ldots & \ldots & \ldots & \ldots & \ldots & \ldots & \ldots & \ldots & \mathrm{x} & \cdots & \ldots & \ldots & \ldots & \ldots & \ldots & \ldots & \ldots & \ldots & \ldots\end{array}$

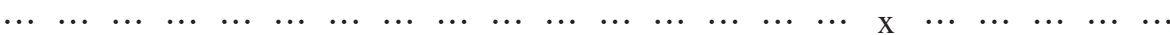

$\begin{array}{lllllllllllllllllllllllll}\ldots & \ldots & \ldots & \ldots & \ldots & \ldots & \ldots & \ldots & \ldots & \ldots & \ldots & \ldots & \ldots & \ldots & \ldots & \cdots & \mathrm{cf} & \ldots & \ldots & \ldots & \ldots & \ldots\end{array}$

$\begin{array}{lllllllllllllllllllllll}\ldots & \ldots & \ldots & \ldots & \ldots & \ldots & \ldots & \ldots & \ldots & \ldots & \ldots & \ldots & \ldots & \ldots & \ldots & \cdots & \mathrm{x} & \ldots & \ldots & \ldots & \ldots & \ldots\end{array}$

$\begin{array}{llllllllllllllllllllll}\ldots & \ldots & \ldots & \ldots & \ldots & \ldots & \ldots & \ldots & \ldots & \ldots & \ldots & \ldots & \ldots & \ldots & \ldots & \cdots & \mathrm{x} & \ldots & \ldots & \ldots & \ldots & \cdots\end{array}$

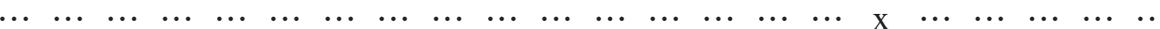

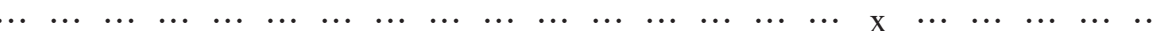

$\begin{array}{lllllllllllllllllllllll} & \ldots & \ldots & \ldots & \ldots & \ldots & \ldots & \ldots & \ldots & \ldots & \ldots & \ldots & \ldots & \ldots & \ldots & \ldots & \ldots & \mathrm{cf} & \ldots & \ldots & \ldots & \ldots & \ldots\end{array}$

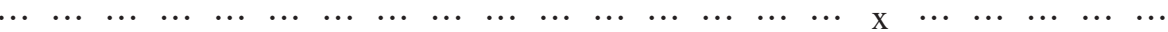

$\begin{array}{lllllllllllllllllllllll}\ldots & \ldots & \ldots & \ldots & \ldots & \ldots & \ldots & \ldots & \ldots & \ldots & \ldots & \ldots & \ldots & \ldots & \ldots & \ldots & \mathrm{cf} & \ldots & \ldots & \ldots & \ldots & \ldots\end{array}$

$\begin{array}{lllllllllllllllllllllll}\mathrm{x} & \cdots & \mathrm{x} & \cdots & \mathrm{x} & \mathrm{x} & \cdots & \cdots & \cdots & \cdots & \mathrm{x} & \mathrm{x} & \mathrm{x} & \cdots & \cdots & \mathrm{x} & \mathrm{x} & \cdots & \cdots & \cdots & \cdots & \cdots\end{array}$

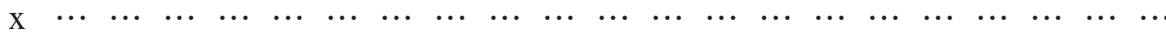

$\begin{array}{llllllllllllllllllllll}\mathrm{x} & \cdots & \mathrm{x} & \cdots & \mathrm{x} & \mathrm{x} & \cdots & \cdots & \cdots & \cdots & \cdots & \cdots & \cdots & \cdots & \cdots & \cdots & \cdots & \cdots & \cdots & \cdots & \cdots & \cdots\end{array}$

$\begin{array}{llllllllllllllllllllll}\ldots & \ldots & \ldots & \ldots & \ldots & \ldots & \ldots & \ldots & \ldots & \ldots & \ldots & \ldots & \ldots & \ldots & \ldots & \cdots & \mathrm{x} & \ldots & \ldots & \ldots & \ldots & \ldots\end{array}$

$\begin{array}{lllllllllllllllllllllll}\cdots & \cdots & \ldots & \ldots & \ldots & \ldots & \ldots & \ldots & \ldots & \cdots & \cdots & \cdots & \cdots & \cdots & \cdots & \mathrm{x} & \cdots & \cdots & \cdots & \cdots & \cdots & \cdots\end{array}$

$\begin{array}{llllllllllllllllllllll}\mathrm{x} & \cdots & \cdots & \ldots & \ldots & \ldots & \ldots & \ldots & \ldots & \ldots & \ldots & \ldots & \ldots & \ldots & \ldots & \ldots & \ldots & \ldots & \ldots & \ldots & \ldots & \ldots\end{array}$

$\begin{array}{llllllllllllllllllllll}\ldots & \ldots & \ldots & \ldots & \ldots & \ldots & \ldots & \ldots & \ldots & \ldots & \ldots & \ldots & \ldots & \ldots & \ldots & \ldots & \ldots & \ldots & \ldots & \ldots & \ldots & \ldots\end{array}$

$\begin{array}{llllllllllllllllllllllll}\ldots & \ldots & \ldots & \ldots & \ldots & \ldots & \ldots & \ldots & \ldots & \ldots & \ldots & \ldots & \ldots & \ldots & \ldots & \ldots & \ldots & \ldots & \ldots & \ldots & \ldots & \ldots\end{array}$

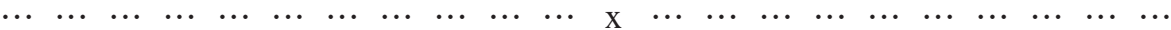

$\begin{array}{lllllllllllllllllllllll}\cdots & \cdots & \mathrm{x} & \cdots & \cdots & \cdots & \cdots & \mathrm{x} & \cdots & \cdots & \mathrm{x} & \cdots & \mathrm{x} & \cdots & \cdots & \mathrm{x} & \cdots & \cdots & \cdots & \cdots & \cdots & \cdots\end{array}$

$\begin{array}{llllllllllllllllllllllll}\cdots & \cdots & \mathrm{x} & \cdots & \cdots & \cdots & \cdots & \cdots & \cdots & \cdots & \mathrm{x} & \cdots & \mathrm{x} & \cdots & \cdots & \mathrm{x} & \cdots & \cdots & \cdots & \cdots & \cdots & \cdots\end{array}$

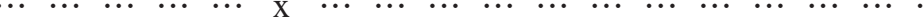

$\begin{array}{lllllllllllllllllllllllllllllll}\mathrm{x} & \cdots & \mathrm{x} & \mathrm{x} & \mathrm{x} & \cdots & \cdots & \cdots & \mathrm{x} & \mathrm{x} & \mathrm{x} & \mathrm{x} & \cdots & \cdots & \mathrm{x} & \mathrm{x} & \cdots & \mathrm{x} & \cdots & \cdots & \cdots\end{array}$

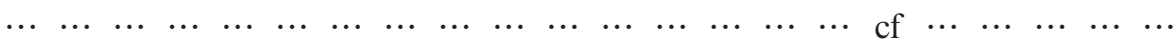

$\begin{array}{llllllllllllllllllllll}\cdots & \cdots & \cdots & \cdots & \cdots & \cdots & \cdots & \cdots & \cdots & \mathrm{x} & \cdots & \cdots & \mathrm{x} & \cdots & \cdots & \cdots & \cdots & \cdots & \cdots & \cdots & \cdots & \cdots\end{array}$

$\begin{array}{llllllllllllllllllllll}\cdots & \cdots & \cdots & \cdots & \cdots & \cdots & \cdots & \cdots & \cdots & \mathrm{x} & \cdots & \cdots & \mathrm{x} & \cdots & \cdots & \cdots & \cdots & \cdots & \cdots & \cdots & \cdots & \cdots\end{array}$

$\begin{array}{llllllllllllllllllllll}\ldots & \ldots & \ldots & \ldots & \ldots & \ldots & \ldots & \ldots & \cdots & \mathrm{x} & \cdots & \ldots & \ldots & \ldots & \ldots & \ldots & \ldots & \ldots & \ldots & \ldots & \ldots & \ldots\end{array}$

$\begin{array}{llllllllllllllllllllllllll}\cdots & \cdots & \cdots & \cdots & \cdots & \cdots & \cdots & \cdots & \cdots & \mathrm{x} & \cdots & \cdots & \mathrm{x} & \cdots & \cdots & \cdots & \cdots & \cdots & \cdots & \cdots & \cdots & \cdots\end{array}$

$\begin{array}{lllllllllllllllllllllllll}\ldots & \ldots & \ldots & \ldots & \ldots & \ldots & \ldots & \ldots & \cdots & \mathrm{x} & \cdots & \ldots & \ldots & \ldots & \ldots & \ldots & \ldots & \ldots & \ldots & \ldots & \ldots & \ldots\end{array}$

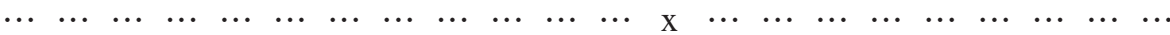

$\begin{array}{llllllllllllllllllllll}\cdots & \cdots & \cdots & \cdots & \cdots & \cdots & \cdots & \cdots & \cdots & \cdots & \cdots & \cdots & \cdots & \cdots & \cdots & \cdots & \text { cf } & \cdots & \cdots & \cdots & \cdots & \cdots\end{array}$

$\begin{array}{llllllllllllllllllllll}\ldots & \ldots & \ldots & \ldots & \ldots & \ldots & \ldots & \ldots & \ldots & \ldots & \ldots & \cdots & \mathrm{x} & \ldots & \ldots & \ldots & \ldots & \ldots & \ldots & \ldots & \ldots & \ldots\end{array}$

$\begin{array}{llllllllllllllllllllll}\cdots & \cdots & \cdots & \mathrm{x} & \cdots & \cdots & \cdots & \cdots & \cdots & \mathrm{x} & \cdots & \cdots & \cdots & \cdots & \cdots & \cdots & \cdots & \cdots & \cdots & \cdots & \ldots & \cdots\end{array}$

$\begin{array}{llllllllllllllllllllll}\cdots & \ldots & \ldots & \ldots & \ldots & \ldots & \ldots & \ldots & \ldots & \ldots & \cdots & \mathrm{x} & \cdots & \cdots & \ldots & \cdots & \cdots & \cdots & \ldots & \ldots & \ldots & \ldots\end{array}$

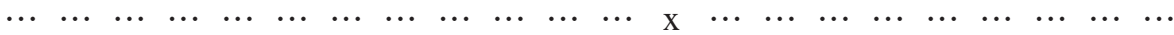

$\begin{array}{llllllllllllllllllllll}\cdots & \cdots & \cdots & \cdots & \cdots & \cdots & \cdots & \cdots & \cdots & \mathrm{x} & \cdots & \cdots & \mathrm{x} & \cdots & \cdots & \cdots & \cdots & \cdots & \cdots & \cdots & \cdots & \cdots\end{array}$ 
Table 1. Continued.

Localities $^{\mathrm{a}}$

Cave Faunas

\begin{tabular}{llllllllllllllllllllll}
\hline 1 & 2 & 3 & 4 & 5 & 6 & 7 & 8 & 9 & 10 & 11 & 12 & 13 & 14 & 15 & 16 & 17 & 18 & 19 & 20 & 21 & 22
\end{tabular}

American widgeon (Anas americana)

Green-winged teal (Anas creca)

Small duck (Anas sp.)

Surface-feeding ducks (Anatini)

Diving ducks (Aythyini)

Common goldeneye (Bucephala clangula)

Goldeneye (Bucephala sp.)

Harlequin duck (Histrionicus histrionicus)

White-winged scoter (Melanitta fusca)

Ruddy duck (Oxyura jamaicensis)

Hooded merganser (Lophodytes cucullatus)

Large merganser (Mergus sp.)

Swans, geese, ducks (Anatidae)

Cooper's hawk (Accipter cooperi)

Red-tailed hawk (Buteo jamaicensis)

Hawk (Buteo sp.)

Hawks and falcons (Falconiformes)

Ruffed grouse (Bonasa umbellus)

Spruce grouse or Ruffed Grouse

(Canachites canadensis or Bonasa umbellus)

Sharp-tailed grouse (Pediocetes phasianellus)

Ptarmigan or grouse (Tetraonidae)

Blue grouse (Dendragrapus obscurus)

Spruce grouse (Canachites canadensis)

Partridge or pheasant or grouse

(Phasianiidae)

Virginia rail or Sora (Rallidae)

Ameircan coot (Fulica americana)

Small wader (Charadriiformes)

American golden plover (Pluvialis dominicalfulva)

Black-bellied plover (Pluvialis squatarola)

Willet (Catoptrophorus semipalmatus)

Eskimo curlew (Numenius borealis)

Solitary sandpiper (Tringa solitaria)

Small Sandpiper (Calidris sp.)

Small gull (Larus sp.)

Small alcid (Alcidae)

Passenger pigeon (Ectopistes

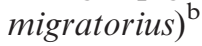

Short-eared owl (Asio flammeus)

Hawk owl (Surnia ulula)
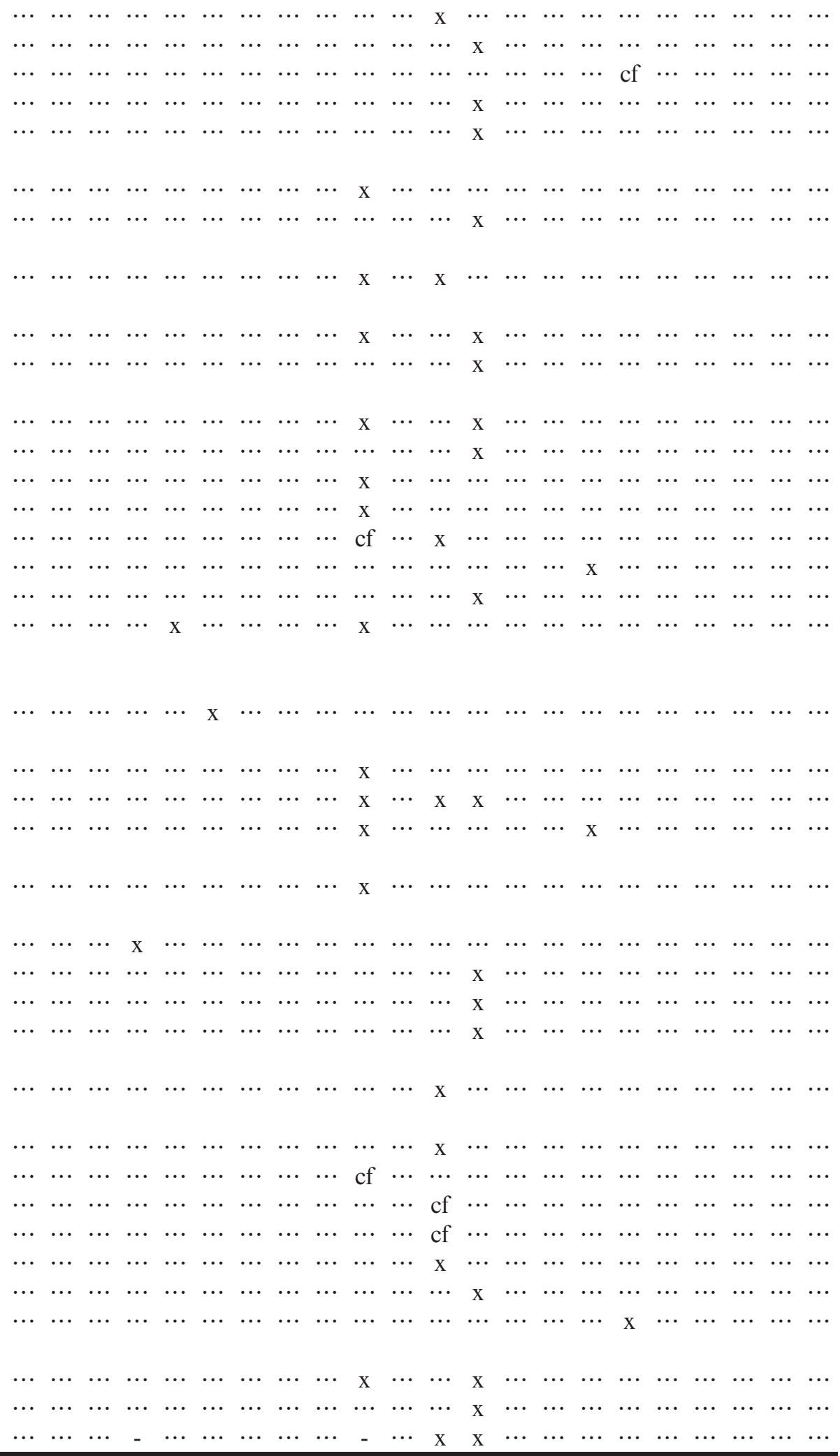
Table 1. Continued.

\section{Localities $^{\mathrm{a}}$}

\begin{tabular}{|c|c|c|c|c|c|c|c|c|c|c|c|c|c|c|c|c|c|c|c|c|c|c|}
\hline Cave Faunas & 1 & 2 & 3 & 4 & 5 & 6 & 7 & 8 & 9 & 10 & 11 & 12 & 13 & 14 & 15 & 16 & 17 & 18 & 19 & 20 & 21 & 22 \\
\hline Snowy owl (Nyctea scandiaca) & . & $\cdot$ & $\cdots$ & $\mathrm{x}$ & $\cdots$ & $\cdots$ & $\cdots$ & $\cdots$ & $\cdots$ & - & $\cdots$ & $\mathrm{x}$ & - & $\cdots$ & . & . & 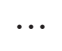 & $\cdots$ & . & . & . & \\
\hline Owls (Strigiformes) & . & $\cdots$ & $\cdots$ & $\cdots$ & $\ldots$ & $\cdots$ & $\cdots$ & $\cdots$ & $\cdots$ & $\mathrm{x}$ & $\cdots$ & $\cdots$ & $\mathrm{x}$ & $\cdots$ & $\cdots$ & $\cdots$ & $\cdots$ & $\cdots$ & $\cdots$ & $\cdots$ & $\cdots$ & $\cdots$ \\
\hline Belted kingfisher (Ceryle alcyon) & & $\cdots$ & $\cdots$ & $\cdots$ & $\cdots$ & $\cdots$ & $\cdots$ & $\cdots$ & $\cdots$ & $\cdots$ & $\cdots$ & $\cdots$ & $\mathrm{x}$ & $\cdots$ & $\cdots$ & $\cdots$ & $\cdots$ & $\cdots$ & $\cdots$ & $\cdots$ & $\cdots$ & $\cdots$ \\
\hline \multicolumn{23}{|l|}{ Hairy woodpecker ( Dendrocopos } \\
\hline villosus) & - & $\cdots$ & $\cdots$ & $\cdots$ & $\cdots$ & $\cdots$ & $\cdots$ & $\cdots$ & $\cdots$ & $\mathrm{X}$ & $\cdots$ & $\cdots$ & $\cdots$ & $\cdots$ & $\cdots$ & $\cdots$ & $\cdots$ & $\cdots$ & $\cdots$ & $\ldots$ & $\cdots$ & $\cdots$ \\
\hline Woodpecker (Picidae) & $\cdots$ & $\cdots$ & $\cdots$ & $\cdots$ & $\cdots$ & $\cdots$ & $\cdots$ & $\cdots$ & $\cdots$ & $\cdots$ & $\cdots$ & $\cdots$ & $\mathrm{x}$ & $\cdots$ & $\cdots$ & $\cdots$ & $\cdots$ & $\cdots$ & $\cdots$ & $\cdots$ & $\cdots$ & $\cdots$ \\
\hline Say's phoebe & . & $\ldots$ & $\cdots$ & $\cdots$ & $\ldots$ & $\cdots$ & $\ldots$ & $\cdots$ & $\ldots$ & $\ldots$ & $\ldots$ & $\mathrm{cf}$ & $\cdots$ & $\ldots$ & $\cdots$ & $\ldots$ & $\ldots$ & $\ldots$ & $\cdots$ & $\ldots$ & $\ldots$ & $\ldots$ \\
\hline \multicolumn{23}{|l|}{ Olive-sided flycatcher (Contopus } \\
\hline contopus) & $\cdots$ & $\cdots$ & $\cdots$ & $\cdots$ & $\cdots$ & $\cdots$ & $\cdots$ & $\cdots$ & $\cdots$ & $\cdots$ & $\cdots$ & $\mathrm{cf}$ & $\cdots$ & $\cdots$ & $\cdots$ & $\cdots$ & $\cdots$ & $\cdots$ & $\cdots$ & $\cdots$ & $\cdots$ & $\cdots$ \\
\hline Barn swallow (Hirundo & $\cdots$ & $\cdots$ & $\cdots$ & $\cdots$ & $\cdots$ & $\cdots$ & $\cdots$ & $\cdots$ & $\cdots$ & $\mathrm{cf}$ & $\cdots$ & $\cdots$ & $\mathrm{cf}$ & $\cdots$ & $\cdots$ & $\cdots$ & $\cdots$ & $\cdots$ & $\cdots$ & $\cdots$ & $\cdots$ & $\cdots$ \\
\hline (Hirundo $p$ & $\ldots$ & $\ldots$ & $\ldots$ & $\ldots$ & $\ldots$ & $\cdots$ & $\ldots$ & $\ldots$ & $\ldots$ & $\mathrm{cf}$ & $\ldots$ & $\ldots$ & $\mathrm{cf}$ & $\ldots$ & $\cdots$ & $\ldots$ & $\ldots$ & $\ldots$ & $\ldots$ & $\ldots$ & ... & $\ldots$ \\
\hline Swallows (Hirundinidae) & $\cdots$ & $\cdots$ & $\cdots$ & $\mathrm{x}$ & $\cdots$ & $\cdots$ & $\ldots$ & $\cdots$ & $\cdots$ & $\cdots$ & $\cdots$ & $\mathrm{x}$ & $\cdots$ & $\cdots$ & $\cdots$ & $\cdots$ & $\cdots$ & $\cdots$ & $\cdots$ & $\cdots$ & $\cdots$ & $\cdots$ \\
\hline Horned lark (Eromophila alpestris) & $\ldots$ & $\ldots$ & $\cdots$ & $\cdots$ & $\ldots$ & $\cdots$ & $\ldots$ & $\ldots$ & $\ldots$ & $\ldots$ & $\ldots$ & $\ldots$ & $\cdots$ & $\cdots$ & $\cdots$ & $\cdots$ & $\mathrm{cf}$ & $\ldots$ & $\ldots$ & $\cdots$ & $\ldots$ & $\ldots$ \\
\hline Blue jay ( $C y a$ & $\mathrm{x}$ & $\cdots$ & $\cdots$ & $\cdots$ & $\cdots$ & $\cdots$ & $\cdots$ & $\cdots$ & $\cdots$ & $\cdots$ & $\cdots$ & $\cdots$ & $\cdots$ & $\cdots$ & $\cdots$ & $\cdots$ & $\cdots$ & $\cdots$ & $\cdots$ & $\cdots$ & $\cdots$ & $\cdots$ \\
\hline Black-billed magpie (Pica pica) & $\cdots$ & $\cdots$ & $\cdots$ & $\cdots$ & $\cdots$ & $\cdots$ & $\cdots$ & $\cdots$ & $\cdots$ & $\mathrm{x}$ & $\cdots$ & $\cdots$ & $\cdots$ & $\cdots$ & $\cdots$ & $\cdots$ & $\cdots$ & $\cdots$ & $\cdots$ & $\cdots$ & $\cdots$ & $\cdots$ \\
\hline \multicolumn{23}{|l|}{ Clark's nutcracker (Nucifraga } \\
\hline columbina) & $\cdots$ & $\cdots$ & $\cdots$ & $\cdots$ & $\cdots$ & $\cdots$ & $\cdots$ & $\cdots$ & $\cdots$ & $\mathrm{X}$ & $\cdots$ & $\cdots$ & $\cdots$ & $\cdots$ & $\cdots$ & $\cdots$ & $\cdots$ & $\cdots$ & $\cdots$ & $\cdots$ & $\cdots$ & $\cdots$ \\
\hline Common ra & $\ldots$ & $\ldots$ & $\cdots$ & $\ldots$ & $\ldots$ & $\cdots$ & $\ldots$ & $\ldots$ & $\cdots$ & $\ldots$ & $\ldots$ & $\mathrm{x}$ & $\mathrm{x}$ & $\ldots$ & $\cdots$ & $\ldots$ & $\ldots$ & $\ldots$ & $\ldots$ & $\ldots$ & $\ldots$ & $\ldots$ \\
\hline Chickadee (Parus sp.) & $\cdots$ & $\cdots$ & $\cdots$ & $\cdots$ & $\cdots$ & $\cdots$ & $\cdots$ & $\cdots$ & $\cdots$ & $\cdots$ & $\cdots$ & $\mathrm{x}$ & $\cdots$ & $\cdots$ & $\cdots$ & $\cdots$ & $\cdots$ & $\cdots$ & $\cdots$ & $\cdots$ & $\cdots$ & $\cdots$ \\
\hline \multicolumn{23}{|l|}{ Red-breasted nuthatch (Sitta } \\
\hline canadensis) & $\mathrm{X}$ & $\cdots$ & $\cdots$ & $\cdots$ & $\cdots$ & $\cdots$ & $\cdots$ & $\cdots$ & $\cdots$ & $\cdots$ & $\cdots$ & $\cdots$ & $\cdots$ & $\cdots$ & $\cdots$ & $\cdots$ & $\cdots$ & $\cdots$ & $\cdots$ & $\cdots$ & $\cdots$ & $\cdots$ \\
\hline Robin (Turdus migratorius) & $\cdots$ & $\cdots$ & $\cdots$ & $\cdots$ & $\cdots$ & $\cdots$ & $\cdots$ & $\ldots$ & $\cdots$ & $\ldots$ & $\cdots$ & $\mathrm{x}$ & $\cdots$ & $\cdots$ & $\cdots$ & $\cdots$ & $\cdots$ & $\cdots$ & $\cdots$ & $\cdots$ & $\cdots$ & $\cdots$ \\
\hline \multicolumn{23}{|l|}{ Hermit thrush or Gray-cheeked } \\
\hline $\mathrm{b}$ (Hvlocichla outtatalmin & $\cdots$ & $\cdots$ & $\cdots$ & $\cdots$ & $\cdots$ & $\cdots$ & $\cdots$ & $\cdots$ & $\cdots$ & $\cdots$ & $\cdots$ & $\mathrm{x}$ & $\cdots$ & $\cdots$ & $\cdots$ & $\cdots$ & $\cdots$ & $\cdots$ & $\cdots$ & $\cdots$ & $\cdots$ & $\cdots$ \\
\hline Waxwing (Bombycilla sp.) & $\cdots$ & $\cdots$ & $\cdots$ & $\cdots$ & $\cdots$ & $\cdots$ & $\cdots$ & $\cdots$ & $\cdots$ & $\cdots$ & $\cdots$ & $\mathrm{x}$ & $\cdots$ & $\cdots$ & $\cdots$ & $\cdots$ & $\cdots$ & $\cdots$ & $\cdots$ & $\cdots$ & $\cdots$ & $\cdots$ \\
\hline \multicolumn{23}{|l|}{ Common redpoll or Hoary redpoll } \\
\hline (Acanthis flammealhornemanni) & . & $\cdots$ & $\cdots$ & $\cdots$ & $\cdots$ & $\cdots$ & $\cdots$ & $\cdots$ & $\cdots$ & $\cdots$ & $\cdots$ & $\mathrm{x}$ & $\cdots$ & $\cdots$ & $\cdots$ & 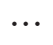 & $\cdots$ & $\cdots$ & $\cdots$ & $\cdots$ & $\cdots$ & $\cdots$ \\
\hline \multicolumn{23}{|l|}{ White-winged crossbill (Loxia } \\
\hline leucoptera) & $\mathrm{cf}$ & 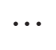 & $\cdots$ & $\cdots$ & $\cdots$ & $\cdots$ & $\cdots$ & $\cdots$ & $\cdots$ & $\cdots$ & $\cdots$ & $\cdots$ & $\cdots$ & $\cdots$ & $\cdots$ & $\mathrm{x}$ & $\cdots$ & $\cdots$ & .. & $\cdots$ & $\cdots$ & $\cdots$ \\
\hline \multicolumn{23}{|l|}{ Savannah sparrow (Passerculus } \\
\hline sandwichensis) & • & $\cdots$ & $\cdots$ & $\cdots$ & $\cdots$ & $\cdots$ & $\cdots$ & $\cdots$ & $\cdots$ & $\cdots$ & $\cdots$ & $\cdots$ & $\cdots$ & $\cdots$ & $\cdots$ & $\mathrm{x}$ & .. & $\cdots$ & $\cdots$ & $\cdots$ & $\cdots$ & $\cdots$ \\
\hline \multicolumn{22}{|l|}{ Tree sparrow or Chipping sparrow } & $\cdots$ \\
\hline \multicolumn{23}{|l|}{ Lincoln's sparrow (Melospiza } \\
\hline & • & $\cdots$ & $\cdots$ & $\cdots$ & $\cdots$ & $\cdots$ & $\cdots$ & $\cdots$ & $\cdots$ & $\cdots$ & $\cdots$ & $\mathrm{cf}$ & $\cdots$ & $\cdots$ & $\cdots$ & $\cdots$ & $\cdots$ & $\cdots$ & $\cdots$ & $\cdots$ & $\cdots$ & $\cdots$ \\
\hline Snc & $\cdots$ & $\cdots$ & $\cdots$ & $\cdots$ & $\cdots$ & $\cdots$ & $\cdots$ & $\cdots$ & $\cdots$ & $\cdots$ & $\cdots$ & $\mathrm{X}$ & $\cdots$ & $\cdots$ & $\cdots$ & $\cdots$ & $\cdots$ & $\cdots$ & $\cdots$ & $\cdots$ & $\cdots$ & $\cdots$ \\
\hline Perching & $\cdots$ & $\cdots$ & $\cdots$ & $\cdots$ & $\cdots$ & $\cdots$ & $\cdots$ & $\cdots$ & $\cdots$ & $\cdots$ & $\cdots$ & $\cdots$ & $\mathrm{X}$ & $\cdots$ & $\cdots$ & $\cdots$ & $\cdots$ & $\cdots$ & $\cdots$ & $\cdots$ & $\cdots$ & $\cdots$ \\
\hline \multirow{3}{*}{\multicolumn{23}{|c|}{ Mammals }} \\
\hline & & & & & & & & & & & & & & & & & & & & & & \\
\hline & & & & & & & & & & & & & & & & & & & & & & \\
\hline Gaspé & $\mathrm{x}$ & $\cdots$ & $\cdots$ & $\cdots$ & $\cdots$ & $\cdots$ & $\cdots$ & $\cdots$ & - & $\cdots$ & $\cdots$ & $\ldots$ & $\ldots$ & $\ldots$ & $\ldots$ & $\ldots$ & $\ldots$ & $\ldots$ & $\ldots$ & $\ldots$ & $\ldots$ & $\ldots$ \\
\hline shrew (Sorex hoyi) & $\mathrm{x}$ & $\cdots$ & $\cdots$ & $\cdots$ & $\cdots$ & $\cdots$ & $\cdots$ & $\cdots$ & $\mathrm{x}$ & $\ldots$ & $\cdots$ & $\cdots$ & $\cdots$ & $\cdots$ & $\cdots$ & $\cdots$ & $\cdots$ & $\cdots$ & $\cdots$ & $\cdots$ & $\cdots$ & $\cdots$ \\
\hline Dusky shrew (Sorex monticollus) & - & $\cdots$ & $\cdots$ & $\cdots$ & $\cdots$ & $\cdots$ & $\cdots$ & $\cdots$ & $\mathrm{x}$ & $\cdots$ & $\mathrm{x}$ & $\cdots$ & $\cdots$ & $\cdots$ & $\cdots$ & $\mathrm{cf}$ & $\cdots$ & $\cdots$ & $\cdots$ & $\cdots$ & $\cdots$ & $\cdots$ \\
\hline \multicolumn{23}{|l|}{ Short-tailed shrew (Blarina } \\
\hline . & & . & $\mathrm{x}$ & $\mathrm{x}$ & $X$ & . & . & $\mathrm{x}$ & $\cdots$ & $\cdots$ & $\ldots$ & $\cdots$ & $\cdots$ & $\cdots$ & $\cdots$ & $\cdots$ & $\cdots$ & $\ldots$ & $\cdots$ & $\ldots$ & $\ldots$ & .. \\
\hline orex sp) & 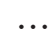 & $\ldots$ & $\cdots$ & $\cdots$ & $\cdots$ & $\cdots$ & $\ldots$ & $\ldots$ & $\mathrm{x}$ & $\ldots$ & $\ldots$ & $\ldots$ & $\ldots$ & $\ldots$ & $\cdots$ & $\mathrm{x}$ & $\cdots$ & $\ldots$ & $\ldots$ & $\ldots$ & $\ldots$ & $\cdots$ \\
\hline Shrew (Soricidae) & $\cdots$ & $\cdots$ & $\cdots$ & $\cdots$ & $\ldots$ & $\cdots$ & $\cdots$ & $\cdots$ & $\cdots$ & $\cdots$ & $\ldots$ & $\mathrm{x}$ & $\cdots$ & $\cdots$ & $\cdots$ & $\cdots$ & $\cdots$ & $\cdots$ & .. & $\ldots$ & $\cdots$ & .. \\
\hline \multicolumn{23}{|l|}{ Hairy-tailed mole (Parascalops } \\
\hline & 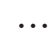 & $\ldots$ & $\mathrm{X}$ & $\ldots$ & $\ldots$ & .. & $\ldots$ & $\ldots$ & $\ldots$ & $\ldots$ & $\ldots$ & $\ldots$ & $\ldots$ & $\cdots$ & $\cdots$ & ... & $\ldots$ & $\ldots$ & .. & $\ldots$ & $\ldots$ & .. \\
\hline
\end{tabular}


Table 1. Continued.

\section{Localities $^{\mathrm{a}}$}

\section{Cave Faunas}

$\begin{array}{llllllllllllllllllllll}1 & 2 & 3 & 4 & 5 & 6 & 7 & 8 & 9 & 10 & 11 & 12 & 13 & 14 & 15 & 16 & 17 & 18 & 19 & 20 & 21 & 22\end{array}$

Star-nosed mole (Condylura cristata)

Little brown bat (Myotis lucifugus)

Keen's bat (Myotis keenii)

Long-legged bat (Myotis volans)

Northern long-eared bat (Myotis septentrionalis)

Bat (Myotis sp.)

Silver-haired bat (Lasionycterus noctivagans)

Big brown bat (Eptesicus fuscus)

Eastern pipistrelle (Pipistrellus subflavus)

Hoary bat (Lasiurus cinereus)

Humans - indirect evidence (Homo sapiens)

American pika (Ochotona princeps)

Large pika (Ochotona sp.)

Eastern cottontail (Sylvilagus floridanus)

Snowshoe hare (Lepus americanus)

Arctic hare (Lepus arcticus)

Large hare (Lepus sp.)

Rabbits and hares (Leporidae)

Eastern chipmunk (Tamias striatus)

Least chipmunk (Eutamias minimus)

Chipmunk (Eutamias sp.)

Woodchuck (Marmota monax)

Hoary marmot (Marmota caligata)

Vancouver Island marmot (Marmota vancouverensis)

Marmot (Marmota sp.)

Columbian ground squirrel

(Spermophilus columbianus)

Golden-mantled ground squirrel (Spermophilus lateralis)

Arctic ground squirrel (Spermophilus parryii)

Ground squirrel (Spermophilus sp.)

Niobrara prairie dog (Cynomys niobrarensis)

Red squirrel (Tamiasciurus hudsonicus)

Tree squirrel (Tamiasciurus sp.)

Northern flying squirrel (Glaucomys sabrinus)

Northern pocket gopher (Thomomys talpoides)

North American beaver (Castor canadensis)

Woodland deer mouse (Peromyscus maniculatus)
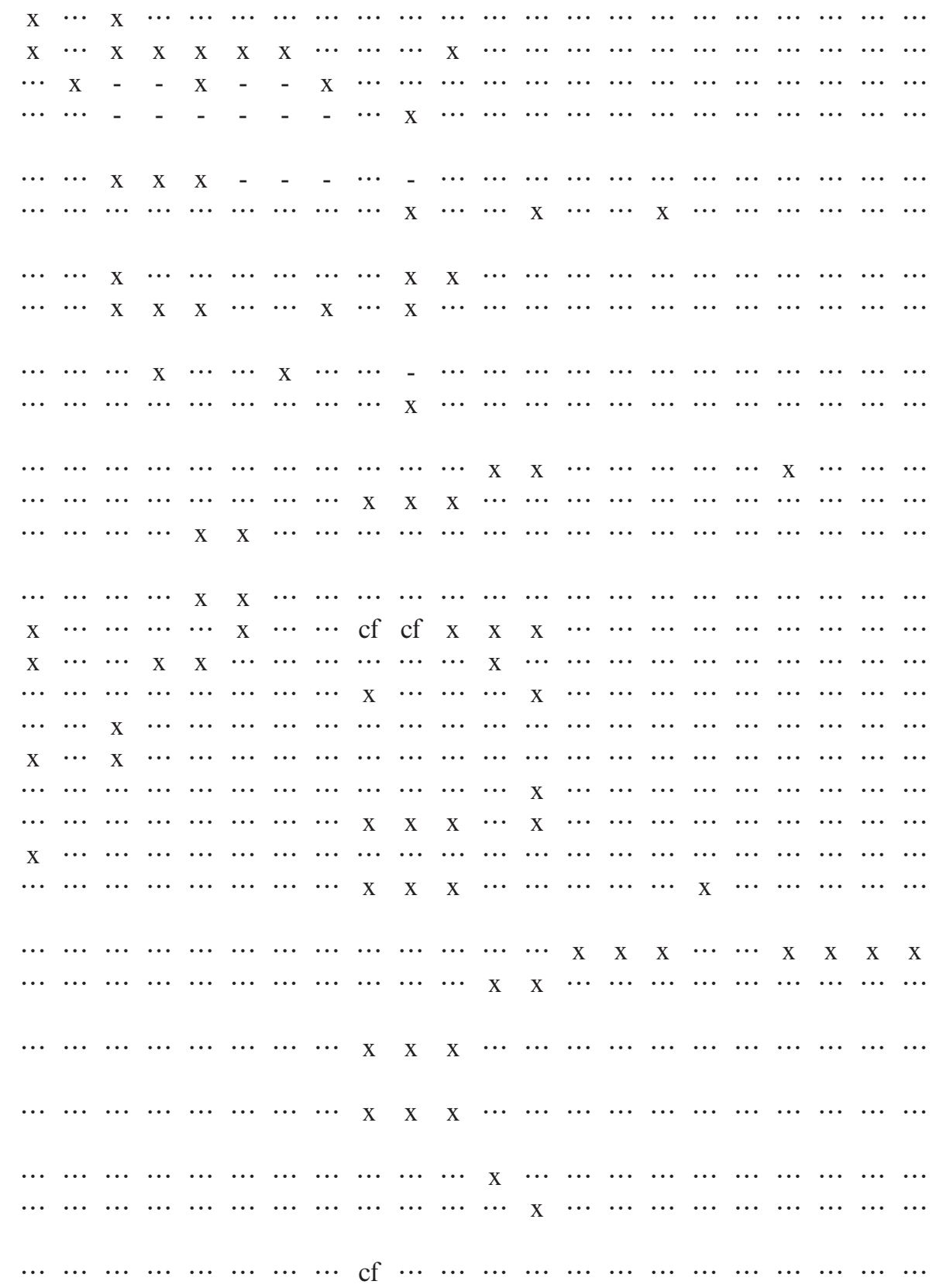

$\begin{array}{llllllllllllllllllllll}\mathrm{x} & \cdots & \mathrm{x} & \mathrm{x} & \mathrm{x} & \cdots & \cdots & \mathrm{x} & \mathrm{x} & \mathrm{x} & \mathrm{x} & \cdots & \cdots & \cdots & \cdots & \mathrm{x} & \cdots & \cdots & \cdots & \cdots & \cdots & \mathrm{x}\end{array}$

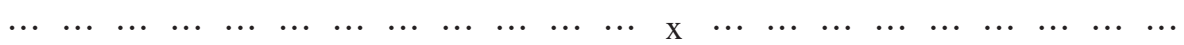

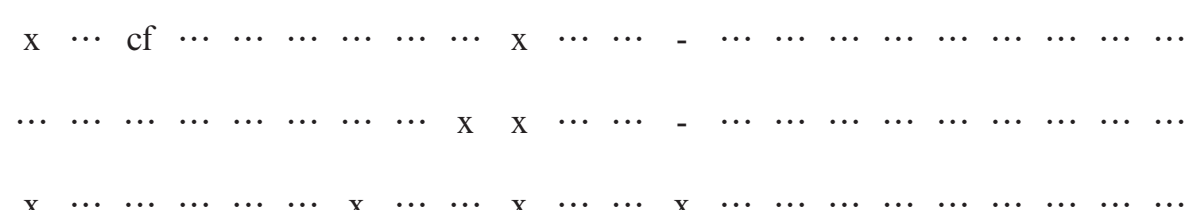
\begin{tabular}{lllllllllllllllllllllll}
$\mathrm{X}$ & $\cdots$ & $\cdots$ & $\cdots$ & $\mathrm{X}$ & $\mathrm{X}$ & $\cdots$ & $\mathrm{X}$ & $\mathrm{X}$ & $\mathrm{X}$ & $\mathrm{X}$ & $\cdots$ & $\cdots$ & $\cdots$ & $\mathrm{X}$ & $\cdots$ & $\cdots$ & $\cdots$ & $\cdots$ & $\cdots$ & $\cdots$ & $\cdots$ \\
\hline
\end{tabular}

168 - Journal of Cave and Karst Studies, December 2011 
Table 1. Continued.

Localities $^{\mathrm{a}}$

\begin{tabular}{|c|c|c|c|c|c|c|c|c|c|c|c|c|c|c|c|c|c|c|c|c|c|c|}
\hline Cave Faunas & 1 & 2 & 3 & 4 & 5 & 6 & 7 & 8 & 9 & 10 & 11 & 12 & 13 & 14 & 15 & 16 & 17 & 18 & 19 & 20 & 21 & 22 \\
\hline Mouse (Peromyscus sp.) & & & $\mathrm{x}$ & $\mathrm{X}$ & $\cdots$ & $\ldots$ & $\ldots$ & $\ldots$ & $\ldots$ & . & $\ldots$ & & $\mathrm{x}$ & & 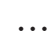 & $\mathrm{x}$ & $\mathrm{x}$ & $\cdots$ & 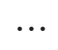 & 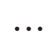 & .. & $\cdots$ \\
\hline Bushy-tailec & & & & & & & & & & & & & & & & & & & & & & \\
\hline $\begin{array}{l}\text { cinerea) } \\
\text { Northern red-backed vole (Myodes }\end{array}$ & $\cdots$ & · & · & $\cdots$ & .. & $\cdots$ & $\cdots$ & $\cdots$ & $\mathrm{x}$ & $\mathrm{x}$ & $\mathrm{x}$ & $\cdots$ & $\mathrm{x}$ & & $\cdots$ & $\cdots$ & $\cdots$ & $\cdots$ & $\cdots$ & $\cdots$ & $\cdots$ & $\cdots$ \\
\hline (Clethrionomys) rutilus) & . & $\ldots$ & $\ldots$ & .. & .. & $\ldots$ & $\ldots$ & $\ldots$ & $\ldots$ & $\ldots$ & $\mathrm{x}$ & $\mathrm{x}$ & $\cdots$ & $\ldots$ & $\ldots$ & $\ldots$ & $\ldots$ & $\ldots$ & $\ldots$ & $\ldots$ & $\ldots$ & $\ldots$ \\
\hline $\begin{array}{l}\text { Southern red-backed vole ( } M \\
\text { (Clethrionomys) gapperi) }\end{array}$ & $\mathrm{X}$ & $\cdots$ & $\mathrm{cf}$ & $\cdots$ & $\cdots$ & $\cdots$ & $\cdots$ & $\mathrm{X}$ & $\mathrm{X}$ & $\mathrm{X}$ & $\cdots$ & $\cdots$ & $\mathrm{X}$ & $\cdots$ & $\cdots$ & $\cdots$ & $\cdots$ & $\cdots$ & $\cdots$ & $\cdots$ & $\cdots$ & $\cdots$ \\
\hline Red-backed v & & & & & & & & & & & & & & & & & & & & & & \\
\hline (Clethriono & $\ldots$ & $\ldots$ & $\ldots$ & $\mathrm{x}$ & $\mathrm{x}$ & $\ldots$ & $\ldots$ & $\ldots$ & $\ldots$ & $\ldots$ & $\ldots$ & $\ldots$ & $\ldots$ & $\ldots$ & $\ldots$ & $\ldots$ & $\ldots$ & $\ldots$ & $\ldots$ & $\ldots$ & $\ldots$ & $\ldots$ \\
\hline Brown lemming (Lemmus sibiricus) & $\cdots$ & $\cdots$ & $\cdots$ & $\cdots$ & $\cdots$ & $\cdots$ & $\cdots$ & $\cdots$ & $\mathrm{x}$ & $\cdots$ & $\cdots$ & $\mathrm{x}$ & $\cdots$ & $\cdots$ & $\cdots$ & $\cdots$ & $\cdots$ & $\cdots$ & $\cdots$ & $\cdots$ & $\cdots$ & $\cdots$ \\
\hline Southern bog len & & & & & & & & & & & & & & & & & & & & & & \\
\hline cooperi) & $\mathrm{x}$ & $\cdots$ & $\mathrm{X}$ & $\cdots$ & $\cdots$ & $\cdots$ & $\cdots$ & $\cdots$ & $\cdots$ & $\cdots$ & $\cdots$ & $\cdots$ & $\cdots$ & $\cdots$ & $\cdots$ & $\cdots$ & $\cdots$ & $\cdots$ & $\cdots$ & $\cdots$ & $\cdots$ & $\cdots$ \\
\hline $\begin{array}{l}\text { Northern bog lemming (Syn } \\
\text { borealis) }\end{array}$ & $\mathrm{x}$ & $\cdots$ & $\cdots$ & $\ldots$ & $\cdots$ & $\cdots$ & $\cdots$ & $\cdots$ & $\mathrm{X}$ & $\cdots$ & $\cdots$ & $\mathrm{x}$ & .. & $\cdots$ & $\cdots$ & $\cdots$ & $\cdots$ & $\cdots$ & $\ldots$ & $\cdots$ & $\cdots$ & $\cdots$ \\
\hline $\begin{array}{l}\text { Western heather vole (Phenacomys } \\
\text { intermedius) }\end{array}$ & & . & & $\mathrm{X}$ & & 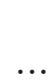 & • & & 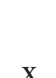 & & & & & & & $\mathrm{x}$ & $\mathrm{x}$ & & & & & \\
\hline Collared lemming (Dicrostonyx & & & & & & & & & 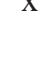 & $X$ & $x$ & & & & & $X$ & $X$ & & & & & \\
\hline torquatus) & $\cdots$ & $\cdots$ & $\cdots$ & .. & $\cdots$ & $\cdots$ & $\cdots$ & $\cdots$ & $\mathrm{x}$ & $\mathrm{x}$ & $\cdots$ & $\mathrm{x}$ & $\mathrm{x}$ & .. & $\cdots$ & $\cdots$ & $\cdots$ & $\cdots$ & $\cdots$ & $\cdots$ & $\cdots$ & $\cdots$ \\
\hline $\begin{array}{l}\text { Ungava coll } \\
\text { (Dicroston }\end{array}$ & 2 & $\mathrm{X}$ & $\mathrm{X}$ & $\mathrm{X}$ & $\cdots$ & $\cdots$ & $\cdots$ & $\cdots$ & $\cdots$ & $\cdots$ & $\cdots$ & $\cdots$ & $\cdots$ & $\cdots$ & $\cdots$ & $\cdots$ & $\cdots$ & $\cdots$ & $\cdots$ & $\ldots$ & $\cdots$ & $\cdots$ \\
\hline Ondatra zibethicus) & $\cdots$ & $\ldots$ & $\mathrm{X}$ & - & - & $\mathrm{x}$ & $\mathrm{x}$ & $\mathrm{x}$ & $\cdots$ & $\mathrm{X}$ & $\mathrm{X}$ & $\mathrm{X}$ & $\mathrm{X}$ & $\cdots$ & .. & $\ldots$ & $\ldots$ & ... & $\cdots$ & $\cdots$ & $\cdots$ & $\cdots$ \\
\hline Sagebrush vole (Lagurus $c$ & .. & $\ldots$ & $\cdots$ & ... & .. & $\ldots$ & $\ldots$ & $\ldots$ & $\mathrm{cf}$ & $\ldots$ & $\ldots$ & .. & $\ldots$ & $\ldots$ & $\ldots$ & $\ldots$ & $\ldots$ & $\ldots$ & $\ldots$ & $\ldots$ & $\cdots$ & $\ldots$ \\
\hline Water vole (Microtus richardsoni) & .. & $\ldots$ & $\ldots$ & ... & ... & ... & $\ldots$ & $\ldots$ & $\mathrm{x}$ & $\mathrm{X}$ & $\ldots$ & ... & $\ldots$ & $\ldots$ & $\ldots$ & $\ldots$ & $\ldots$ & $\ldots$ & $\ldots$ & $\ldots$ & $\cdots$ & ... \\
\hline Meadow & & & & & & & & & & & & & & & & & & & & & & \\
\hline & $\mathrm{x}$ & $\cdots$ & $\mathrm{x}$ & $\mathrm{x}$ & $\mathrm{x}$ & $\cdots$ & $\cdots$ & $\mathrm{x}$ & $\cdots$ & $\mathrm{x}$ & $\mathrm{x}$ & $\mathrm{x}$ & $\mathrm{x}$ & $\cdots$ & $\cdots$ & $\cdots$ & $\ldots$ & $\cdots$ & $\cdots$ & $\cdots$ & $\cdots$ & $\cdots$ \\
\hline Microtus chrot & $\mathrm{x}$ & $\ldots$ & $\mathrm{cf}$ & ... & ... & $\ldots$ & $\ldots$ & $\ldots$ & $\ldots$ & $\ldots$ & $\ldots$ & ... & $\ldots$ & $\ldots$ & .. & ... & $\ldots$ & ... & .. & $\ldots$ & $\ldots$ & ... \\
\hline Taiga vole (Microtus xanthognathus) & r & $\cdots$ & - & $\cdots$ & $\cdots$ & $\cdots$ & $\cdots$ & $\cdots$ & $\cdots$ & $\ldots$ & $\ldots$ & $\mathrm{x}$ & $\mathrm{x}$ & $\cdots$ & $\cdots$ & $\cdots$ & $\ldots$ & $\ldots$ & $\cdots$ & $\ldots$ & $\ldots$ & .. \\
\hline Woodland vole & $\ldots$ & $\ldots$ & $\mathrm{cf}$ & ... & $\ldots$ & $\ldots$ & $\ldots$ & $\ldots$ & $\ldots$ & $\ldots$ & $\ldots$ & ... & $\ldots$ & $\ldots$ & $\ldots$ & $\ldots$ & $\ldots$ & $\ldots$ & $\ldots$ & $\ldots$ & $\ldots$ & $\ldots$ \\
\hline Long-tai & & & & & & & & & & & & & & & & & & & & & & \\
\hline 1 & $\ldots$ & $\ldots$ & $\ldots$ & ... & $\ldots$ & $\ldots$ & $\ldots$ & $\ldots$ & $\mathrm{cf}$ & $\mathrm{cf}$ & $\ldots$ & $\ldots$ & $\ldots$ & $\ldots$ & $\ldots$ & $\ldots$ & $\mathrm{X}$ & $\ldots$ & $\ldots$ & $\ldots$ & $\ldots$ & $\ldots$ \\
\hline Singing & $\ldots$ & $\ldots$ & $\cdots$ & $\cdots$ & .. & $\ldots$ & $\ldots$ & $\ldots$ & $\ldots$ & $\ldots$ & $\ldots$ & $\mathrm{x}$ & $\ldots$ & $\ldots$ & $\ldots$ & $\ldots$ & $\ldots$ & $\ldots$ & $\ldots$ & $\ldots$ & $\cdots$ & $\ldots$ \\
\hline Tundra vole (Microtus oecon & $\ldots$ & $\ldots$ & $\ldots$ & ... & $\ldots$ & $\ldots$ & $\ldots$ & $\ldots$ & $\ldots$ & $\ldots$ & $\ldots$ & $\mathrm{x}$ & $\ldots$ & $\ldots$ & $\ldots$ & $\ldots$ & $\ldots$ & $\ldots$ & .. & $\ldots$ & $\ldots$ & $\ldots$ \\
\hline Townsend' & & & & & & & & & & & & & & & & & & & & & & \\
\hline & $\cdots$ & $\cdots$ & $\cdots$ & $\cdots$ & $\cdots$ & $\cdots$ & $\ldots$ & $\cdots$ & $\ldots$ & $\ldots$ & $\cdots$ & $\cdots$ & $\ldots$ & $\cdots$ & $\cdots$ & $\cdots$ & $\mathrm{x}$ & $\cdots$ & $\cdots$ & $\cdots$ & $\cdots$ & $\cdots$ \\
\hline $\begin{array}{l}\text { Meadow or long-tailed vole } \\
\text { (Microtus pennsylvanicus/ }\end{array}$ & & & & & & & & & & & & & & & & & & & & & & \\
\hline lon & $\cdots$ & $\ldots$ & $\ldots$ & .. & .. & $\ldots$ & $\ldots$ & $\ldots$ & $\ldots$ & $\ldots$ & $\ldots$ & .. & $\mathrm{x}$ & $\ldots$ & ... & $\ldots$ & $\ldots$ & ... & $\ldots$ & $\ldots$ & $\ldots$ & .. \\
\hline Vole (Microtus sp.) & $\cdots$ & $\cdots$ & $\cdots$ & $\cdots$ & $\cdots$ & $\cdots$ & $\cdots$ & $\cdots$ & $\cdots$ & $\cdots$ & $\cdots$ & $\cdots$ & $\cdots$ & $\cdots$ & $\cdots$ & $\mathrm{x}$ & $\cdots$ & $\cdots$ & $\cdots$ & $\cdots$ & $\cdots$ & $\cdots$ \\
\hline Meadow j & & & & & & & & & & & & & & & & & & & & & & \\
\hline & $\mathrm{x}$ & $\cdots$ & $\cdots$ & $\cdots$ & $\cdots$ & $\cdots$ & $\ldots$ & $\cdots$ & $\ldots$ & $\ldots$ & $\ldots$ & $\cdots$ & $\ldots$ & $\cdots$ & $\cdots$ & $\ldots$ & $\cdots$ & $\ldots$ & $\cdots$ & $\ldots$ & $\cdots$ & .. \\
\hline Woodland jumping mouse & & & & & & & & & & & & & & & & & & & & & & \\
\hline$(N a p$ & $\boldsymbol{A}$ & $\cdots$ & $\cdots$ & $\cdots$ & .. & $\ldots$ & $\cdots$ & $\cdots$ & $\cdots$ & $\cdots$ & $\cdots$ & .. & $\cdots$ & $\cdots$ & $\cdots$ & $\ldots$ & $\cdots$ & .. & $\cdots$ & $\ldots$ & $\ldots$ & ... \\
\hline North American $\mathrm{p}$ & & & & & & & & & & & & & & & & & & & & & & \\
\hline & $\boldsymbol{\lambda}$ & $\mathrm{x}$ & $\mathrm{X}$ & $\cdots$ & $\ldots$ & $\ldots$ & $\ldots$ & $\cdots$ & $\ldots$ & $\ldots$ & $\mathrm{x}$ & .. & $\mathrm{X}$ & $\cdots$ & $\ldots$ & $\ldots$ & $\ldots$ & $\ldots$ & $\cdots$ & $\cdots$ & $\cdots$ & ... \\
\hline Coyote (Canis latrans) & $\ldots$ & $\ldots$ & $\mathrm{cf}$ & ... & $\ldots$ & $\ldots$ & $\ldots$ & $\ldots$ & $\mathrm{x}$ & $\mathrm{x}$ & $\ldots$ & $\ldots$ & $\ldots$ & $\ldots$ & $\ldots$ & $\ldots$ & $\ldots$ & $\ldots$ & $\ldots$ & $\ldots$ & $\ldots$ & ... \\
\hline s lupus) & ... & $\ldots$ & $\ldots$ & .. & $\ldots$ & $\ldots$ & ... & $\ldots$ & $\ldots$ & $\mathrm{x}$ & $\mathrm{x}$ & $\mathrm{x}$ & $\mathrm{x}$ & $\ldots$ & $\cdots$ & $\ldots$ & $\ldots$ & $\ldots$ & $\cdots$ & $\ldots$ & $\cdots$ & .. \\
\hline yote (Canis lupus/latrans) & $\cdots$ & $\cdots$ & $\cdots$ & $\cdots$ & $\cdots$ & $\cdots$ & $\cdots$ & $\cdots$ & $\cdots$ & $\cdots$ & $\mathrm{x}$ & $\cdots$ & $\cdots$ & $\cdots$ & $\cdots$ & $\cdots$ & $\cdots$ & $\cdots$ & $\cdots$ & $\cdots$ & $\cdots$ & ... \\
\hline Wolf or dog (Canis lupus/familiaris) & .. & $\ldots$ & $\cdots$ & ... & ... & ... & $\ldots$ & $\ldots$ & $\ldots$ & $\ldots$ & $\ldots$ & .. & $\mathrm{X}$ & $\ldots$ & .. & $\ldots$ & $\ldots$ & .. & .. & $\cdots$ & $\ldots$ & ... \\
\hline arks (Canidae) & $\ldots$ & $\ldots$ & $\ldots$ & .. & $\ldots$ & $\ldots$ & $\ldots$ & $\ldots$ & $\ldots$ & $\ldots$ & $\ldots$ & .. & $\ldots$ & $\ldots$ & $\ldots$ & $\ldots$ & $\mathrm{cf}$ & $\ldots$ & $\ldots$ & $\ldots$ & $\ldots$ & ... \\
\hline Arctic fox (Alopex lagopus) & $\ldots$ & $\ldots$ & $\ldots$ & $\mathrm{x}$ & $\ldots$ & $\ldots$ & $\ldots$ & $\ldots$ & $\ldots$ & $\ldots$ & $\ldots$ & $\mathrm{x}$ & $\ldots$ & $\ldots$ & $\ldots$ & $\ldots$ & $\ldots$ & $\ldots$ & $\ldots$ & $\ldots$ & $\ldots$ & ... \\
\hline
\end{tabular}


Table 1. Continued.

\section{Localities $^{\mathrm{a}}$}

Cave Faunas

Red fox (Vulpes vulpes)

Swift fox (Vulpes velox)

Fox (Vulpes sp.)

Giant short-faced bear (Arctodus simus $)^{\mathrm{b}}$

Black bear (Ursus americanus)

Brown bear (Ursus arctos)

Bear (Ursus sp.)

Raccoon (Procyon lotor)

Noble marten (Martes americana nobilis) ${ }^{\mathrm{b}}$

American marten (Martes americana)

Fisher (Martes pennanti)

Beringian ferret (Mustela eversmanni) ${ }^{\mathrm{b}}$

Black-footed ferret (Mustela nigripes)

Least weasel (Mustela nivalis)

Ermine (Mustela erminea)

Long-tailed weasel (Mustela frenata)

American mink (Mustela vison)

Weasel (Mustela sp.)

Wolverine (Gulo gulo)

American badger (Taxidea taxus)

Short-faced skunk (Brachyprotoma

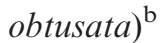

Striped skunk (Mephitis mephitis)

Northern river otter (Lontra canadensis)

Canada lynx (Lynx canadensis)

Bobcat (Lynx rufus)

Cougar (Puma concolor)

Domestic cat (Felis catus)

Steppe lion (Panthera leo spelaea) ${ }^{\mathrm{b}}$

Woolly mammoth (Mammuthus

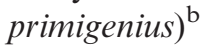

Yukon horse (Equus lambei) ${ }^{\mathrm{b}}$

Horse (Equus sp.)

Caribou (Rangifer tarandus)

Mule deer (Odocoileus hemionus)

Black-tailed deer (Odocoileus

hemionus columbianus)

White-tailed deer (Odocoileus virginianus)

Deer (Odocoileus sp.)

Moose (Alces alces)

Wapiti or elk (Cervus elaphus)

Steppe bison (Bison priscus) ${ }^{\mathrm{b}}$

Bison (Bison sp.)

American bison or cattle (Bison

bison/Bos taurus)

Saiga antelope $(\text { Saiga tatarica })^{\mathrm{b}}$ $\begin{array}{llllllllllllllllllllll}1 & 2 & 3 & 4 & 5 & 6 & 7 & 8 & 9 & 10 & 11 & 12 & 13 & 14 & 15 & 16 & 17 & 18 & 19 & 20 & 21 & 22\end{array}$

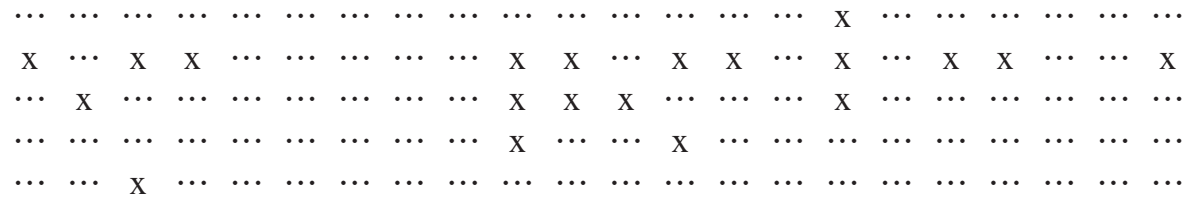

$\begin{array}{llllllllllllllllllllll}\ldots & \ldots & \ldots & \ldots & \ldots & \ldots & \ldots & \ldots & \ldots & \ldots & \ldots & \ldots & \ldots & \ldots & \ldots & \ldots & \mathrm{cf} & \ldots & \ldots & \ldots & \ldots & \ldots\end{array}$

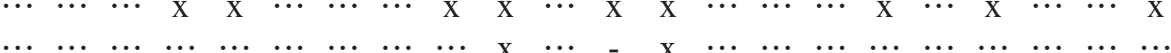

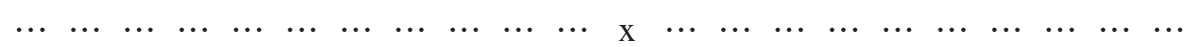

$\begin{array}{llllllllllllllllllllll}\cdots & \cdots & \cdots & \cdots & \cdots & \cdots & \cdots & \cdots & \mathrm{x} & \cdots & \cdots & \cdots & \cdots & \cdots & \cdots & \cdots & \cdots & \cdots & \cdots & \cdots & \cdots & \cdots\end{array}$

$\begin{array}{llllllllllllllllllllll}\mathrm{x} & \cdots & \cdots & \cdots & \cdots & \cdots & \cdots & \cdots & \mathrm{x} & \cdots & \cdots & \mathrm{x} & \mathrm{x} & \cdots & \cdots & \cdots & \cdots & \cdots & \cdots & \cdots & \cdots & \cdots\end{array}$

$\begin{array}{llllllllllllllllllllll}\mathrm{x} & \cdots & \cdots & \mathrm{x} & \cdots & \cdots & \cdots & \cdots & \mathrm{x} & \mathrm{x} & \cdots & \mathrm{x} & \mathrm{x} & \cdots & \cdots & \cdots & \cdots & \cdots & \cdots & \cdots & \cdots & \cdots\end{array}$

$\begin{array}{llllllllllllllllllllll}\cdots & \cdots & \ldots & \ldots & \ldots & \ldots & \ldots & \cdots & \mathrm{x} & \mathrm{x} & \mathrm{x} & \cdots & \cdots & \cdots & \ldots & \ldots & \ldots & \ldots & \ldots & \ldots & \ldots & \ldots\end{array}$

$\begin{array}{lllllllllllllllllllllll}\cdots & \cdots & \cdots & \cdots & \cdots & \cdots & \cdots & \cdots & \cdots & \mathrm{cf} & \mathrm{x} & \cdots & \mathrm{x} & \cdots & \cdots & \cdots & \cdots & \cdots & \cdots & \cdots & \ldots & \cdots\end{array}$

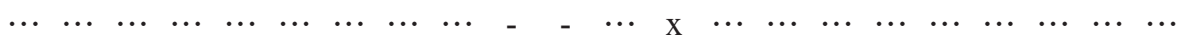

$\begin{array}{llllllllllllllllllllll}\mathrm{x} & \cdots & \cdots & \cdots & \cdots & \cdots & \cdots & \cdots & \cdots & \mathrm{x} & \mathrm{x} & \cdots & \mathrm{x} & \cdots & \cdots & \cdots & \cdots & \cdots & \cdots & \cdots & \cdots & \cdots\end{array}$

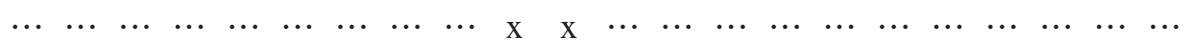

$\begin{array}{llllllllllllllllllllllll}\cdots & \cdots & \cdots & \cdots & \cdots & \cdots & \cdots & \cdots & \cdots & \cdots & \cdots & \mathrm{x} & \cdots & \cdots & \cdots & \cdots & \cdots & \cdots & \cdots & \cdots & \cdots & \cdots\end{array}$

$\begin{array}{lllllllllllllllllllllll}\cdots & \cdots & \cdots & \cdots & \cdots & \mathrm{x} & \cdots & \cdots & \cdots & \mathrm{x} & \cdots & \cdots & \mathrm{x} & \cdots & \cdots & \cdots & \cdots & \cdots & \cdots & \cdots & \cdots & \cdots\end{array}$

$\begin{array}{llllllllllllllllllllll}\cdots & \cdots & \cdots & \cdots & \cdots & \cdots & \cdots & \cdots & \mathrm{x} & \mathrm{x} & \cdots & \cdots & \cdots & \cdots & \cdots & \cdots & \cdots & \cdots & \cdots & \cdots & \cdots & \cdots\end{array}$

$\begin{array}{llllllllllllllllllllll}\cdots & \cdots & \cdots & \cdots & \cdots & \cdots & \cdots & \cdots & \cdots & \mathrm{cf} & \mathrm{x} & \cdots & \mathrm{x} & \cdots & \cdots & \cdots & \cdots & \cdots & \cdots & \cdots & \cdots & \cdots\end{array}$

$\begin{array}{llllllllllllllllllllllll}\ldots & \ldots & \ldots & \ldots & \ldots & \ldots & \ldots & \ldots & \ldots & \mathrm{cf} & \ldots & \ldots & \ldots & \ldots & \ldots & \ldots & \ldots & \ldots & \ldots & \ldots & \ldots & \ldots\end{array}$

$\begin{array}{lllllllllllllllllllllll}\ldots & \cdots & \ldots & \ldots & \ldots & \ldots & \ldots & \ldots & \ldots & \ldots & \cdots & \mathrm{x} & \mathrm{x} & \cdots & \cdots & \cdots & \cdots & \cdots & \cdots & \cdots & \cdots & \cdots\end{array}$

$\begin{array}{llllllllllllllllllllll}\ldots & \ldots & \ldots & \ldots & \ldots & \ldots & \ldots & \ldots & \ldots & \ldots & \cdots & \cdots & \mathrm{x} & \cdots & \cdots & \cdots & \cdots & \cdots & \cdots & \cdots & \ldots & \cdots\end{array}$

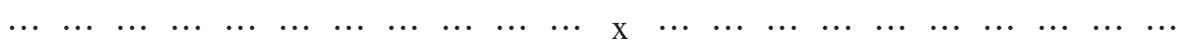

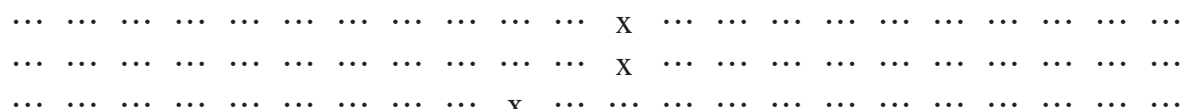

$\begin{array}{llllllllllllllllllllll}\mathrm{x} & \mathrm{x} & \cdots & \mathrm{x} & \cdots & \cdots & \cdots & \cdots & \cdots & \cdots & \cdots & \mathrm{x} & \cdots & \cdots & \cdots & \cdots & \cdots & \cdots & \cdots & \cdots & \cdots & \cdots\end{array}$

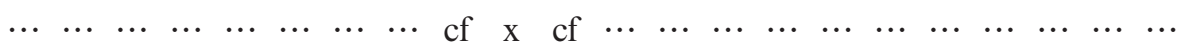

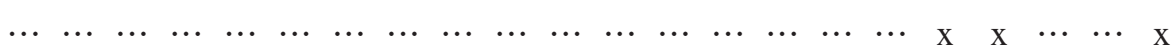

$\begin{array}{llllllllllllllllllllll}\cdots & \cdots & \mathrm{x} & \cdots & \mathrm{x} & \cdots & \mathrm{x} & \cdots & \cdots & \cdots & \cdots & \cdots & \cdots & \cdots & \cdots & \cdots & \cdots & \cdots & \cdots & \cdots & \cdots & \cdots\end{array}$

$\begin{array}{llllllllllllllllllllllll}\cdots & \cdots & \cdots & \cdots & \cdots & \cdots & \cdots & \cdots & \cdots & \cdots & \cdots & \cdots & \mathrm{x} & \cdots & \cdots & \mathrm{x} & \cdots & \cdots & \cdots & \cdots & \cdots & \cdots\end{array}$

$\begin{array}{llllllllllllllllllllllllll}\mathrm{x} & \mathrm{x} & \cdots & \cdots & \cdots & \cdots & \cdots & \cdots & \cdots & \cdots & \cdots & \mathrm{x} & \mathrm{x} & \cdots & \cdots & \cdots & \cdots & \cdots & \cdots & \cdots & \cdots & \cdots\end{array}$

$\begin{array}{llllllllllllllllllllllll}\cdots & \cdots & \cdots & \cdots & \cdots & \cdots & \cdots & \mathrm{x} & \cdots & \cdots & \mathrm{x} & \mathrm{x} & \mathrm{x} & \cdots & \cdots & \cdots & \cdots & \cdots & \cdots & \cdots & \cdots & \cdots\end{array}$

$\begin{array}{llllllllllllllllllllll}\ldots & \ldots & \ldots & \ldots & \ldots & \ldots & \ldots & \ldots & \ldots & \ldots & \cdots & \mathrm{x} & \cdots & \ldots & \ldots & \ldots & \ldots & \ldots & \ldots & \ldots & \ldots & \ldots\end{array}$

$\begin{array}{llllllllllllllllllllllll}\cdots & \cdots & \cdots & \cdots & \cdots & \cdots & \cdots & \cdots & \cdots & \mathrm{x} & \mathrm{x} & \cdots & \mathrm{x} & \cdots & \cdots & \cdots & \cdots & \cdots & \cdots & \cdots & \cdots & \cdots\end{array}$

$\begin{array}{llllllllllllllllllllll}\ldots & \ldots & \ldots & \ldots & \ldots & \ldots & \ldots & \ldots & \ldots & \mathrm{x} & \ldots & \ldots & \ldots & \ldots & \ldots & \ldots & \ldots & \ldots & \ldots & \ldots & \ldots & \ldots\end{array}$

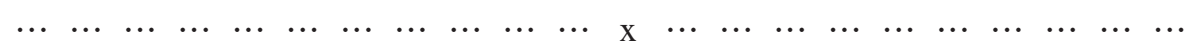


Table 1. Continued.

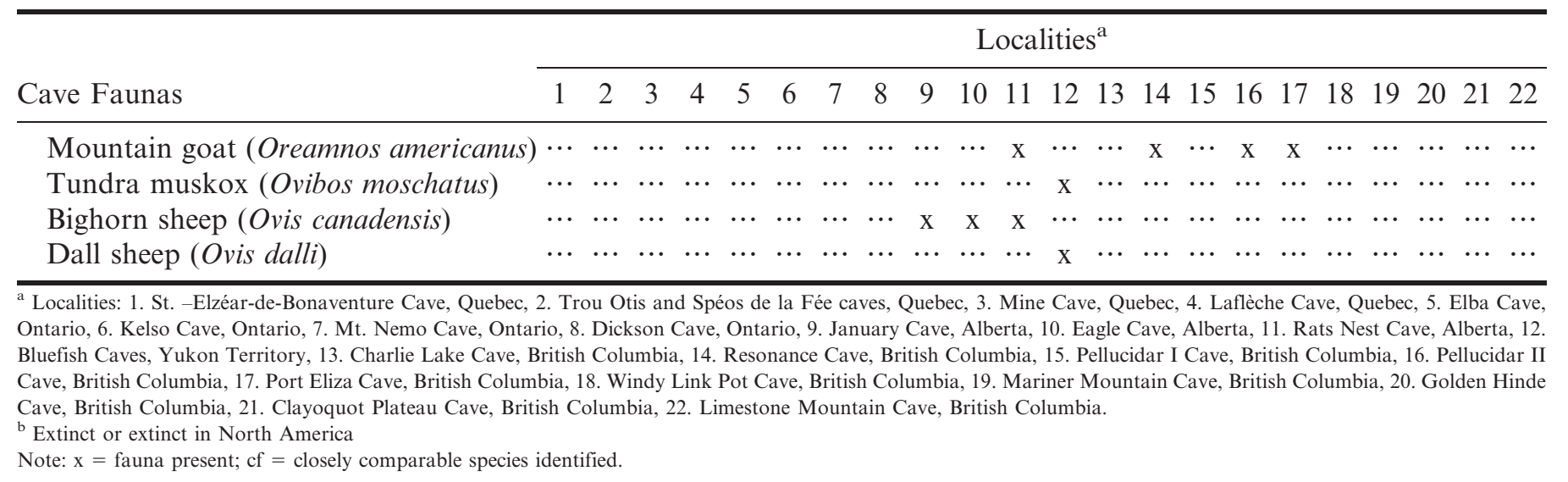

well above its present elevation range in this part of the Rocky Mountains (Burns, 1982; 1987).

Rats Nest Cave, a natural trap on Grotto Mountain near Exshaw, Alberta, yielded remains of fish, amphibians, reptiles, birds, two species of shrew (Sorex arcticus and $S$. monticollus), two species of bat (Lasionycterus noctivagans and Myotis lucifugus), the American pika Ochotona princeps, the snowshoe hare, the hoary marmot, two species of ground squirrel (Spermophilus columbianus and Spermophilus lateralis), the red squirrel, the deer mouse, the wood rat, three species of vole, the muskrat, the North American porcupine, the brown bear, the black bear, the gray wolf Canis lupus, the swift fox Vulpes velox, the American mink Mustela vison, the long-tailed weasel Mustela frenata, the wolverine Gulo gulo, the American badger Taxidea taxus, the Canadian lynx Lynx canadensis, the mule deer, the wapiti or elk, the bighorn sheep, and the mountain goat Oreamnos americanus. Radiocarbon dating of bone gave ages ranging from about 7,000 to 2,500 B.P. (Burns, 1989).

\section{YUKON}

Bluefish Caves (I-III; $67^{\circ} 08^{\prime} \mathrm{N}, 140^{\circ} 47^{\prime} \mathrm{W}$; inset Fig. 1b; Fig. 4a), located $54 \mathrm{~km}$ southwest of the village of Old Crow at an elevation of $250 \mathrm{~m}$, have yielded evidence of episodic human activity from about 25,000 to 10,000 B.P. I consider them to be the most significant of Canadian cave faunas because they contain evidence for the earliest humans (Homo sapiens) in North America, a well-marked transition between Pleistocene and Holocene sediments, flora, and fauna, and well-preserved bones representing a substantial variety of both larger and smaller mammals adapted to northern conditions. An important specimen from Cave II is a mammoth (Mammuthus primigenius) limb-bone flake and its parent core (Fig. 4b) AMS radiocarbon-dated to 23,500 B.P.; both flake and core were dated, the dates overlapping at one sigma, providing that average date. Since the contents of the caves constrain the range of taphonomic processes that could account for the breaking and flaking of a large mammoth bone, the flake and core were produced by humans. Further, a split caribou tibia, reminiscent of a broken fleshing tool, has been dated to 24,800 B.P. The ice-age fauna of Bluefish Caves comprises four fish species, one amphibian, at least twenty-three bird species, and thirty-five mammal species, including the mammoth, the steppe bison Bison priscus, the Yukon horse Equus lambei, the Dall sheep Ovis dalli, the caribou, the moose, the wapiti, the saiga antelope Saiga tatarica, the muskox Ovibos moschatus, the steppe lion Panthera leo spelaea, the cougar Puma concolor, the brown bear, the wolf, and many smaller mammals, including nine species of microtine rodents. Stone artifacts, made of exotic, high-quality cherts, were mainly found in loess that contained remains of the Late Pleistocene vertebrate fauna (Beebe, 1983; Cinq-Mars, 1979; Cinq-Mars and Morlan, 1999; Harington and Cinq-Mars, 2008; Morlan, 1989; McCuaig-Balkwill and Cinq-Mars, 1998).

\section{British Columbia}

A wealth of information about vertebrates of northeastern British Columbia spanning the last 10,800 years has come from Charlie Lake Cave $\left(56^{\circ} 16^{\prime} 35^{\prime \prime} \mathrm{N}, 120^{\circ} 56^{\prime} 15^{\prime \prime} \mathrm{W}\right)$, which is about $7 \mathrm{~km}$ northeast of Fort St. John (Fig. 1b; Fig. 5). Following the retreat of Glacial Lake Peace from this site shortly before 10,500 years ago, the gully in front of the cave began filling with sediment, ultimately reaching a depth of more than $4 \mathrm{~m}$. During this process, the site was visited occasionally by people who left stone artifacts and bones as early as 10,500 B.P. It was also visited by predators carrying their prey and by various animals that lived in the area. The basal unit (about 10,500-9,000 B.P.) produced fossils including remains of suckers Catostomus $\mathrm{sp}$. and other unidentified fishes. Frogs also suggest nearby lakes or streams. Of the birds found, evidently the horned grebe Podiceps auritus, the grouse and ptarmigan of family Tetraonidae, the American coot Fulica americana, the short-eared owl Asio flammeus, and the cliff swallow Hirundo pyrrhonota were most common. Other bird remains collected represent the western grebe Aechmophorus occidentalis, medium-sized grebes of family Podice- 
Table 2. Radiocarbon ages of some vertebrates from Canadian caves. Dates in parentheses are normalized.

\begin{tabular}{|c|c|c|c|c|c|}
\hline Cave & Species & $\begin{array}{l}\text { Radiocarbon } \\
\text { Age }(\mathrm{BP})\end{array}$ & Lab Number ${ }^{\mathrm{a}}$ & Reference & Remarks \\
\hline \multirow{4}{*}{$\begin{array}{l}\text { St. Elzéar Cave, } \\
\text { Quebec }\end{array}$} & Moose (Alces alces) & $410 \pm 120$ & QU-714 & LaSalle 1984 & \\
\hline & Moose (Alces alces) & $4400 \pm 130$ & QU-717 & LaSalle 1984 & \\
\hline & Moose (Alces alces) & $4390 \pm 120$ & QU-745 & LaSalle 1984 & \\
\hline & Moose (Alces alces) & $5110 \pm 150$ & QU-7016 & LaSalle 1984 & \\
\hline \multirow[t]{2}{*}{$\begin{array}{l}\text { Laflèche Cave, } \\
\text { Quebec }\end{array}$} & $\begin{array}{l}\text { Arctic fox (Alopex } \\
\text { lagopus) }\end{array}$ & $(10,800 \pm 90)$ & TO-1197 & Harington $2003 \mathrm{~b}$ & \\
\hline & $\begin{array}{c}\text { Unspecified mammal } \\
\text { bone (Mammalia) }\end{array}$ & $\begin{array}{c}9340 \pm 80 \\
(9310 \pm 80)\end{array}$ & Beta-83094 & Harington 2003b & \\
\hline \multirow[t]{2}{*}{$\begin{array}{c}\text { Elba Cave, } \\
\text { Ontario }\end{array}$} & Pika (Ochotona sp.) & $8670 \pm 220$ & ТO-2566 & $\begin{array}{l}\text { Savage 1994; Mead and } \\
\text { Grady } 1996\end{array}$ & \\
\hline & $\begin{array}{l}\text { American marten } \\
\quad(\text { Martes americana) }\end{array}$ & $510 \pm 60$ & TO- & Savage 1994 & $\begin{array}{l}\text { Lab number is not } \\
\text { recorded. }\end{array}$ \\
\hline $\begin{array}{c}\text { January Cave, } \\
\text { Alberta }\end{array}$ & $\begin{array}{l}\text { Brown lemming } \\
\quad(\text { Lemmus sibiricus })\end{array}$ & $23,100 \pm 860$ & GaK-5438 & Burns 1980 & \\
\hline \multirow[t]{2}{*}{$\begin{array}{l}\text { Eagle Cave, } \\
\text { Alberta }\end{array}$} & $\begin{array}{l}\text { Hoary marmot } \\
\quad(\text { Marmota caligata) }\end{array}$ & $(29,180 \pm 300)$ & TO-6351 & Burns 1991, 1996 & \\
\hline & $\begin{array}{l}\text { Hoary marmot } \\
\quad \text { (Marmota caligata) }\end{array}$ & $(34,860 \pm 470)$ & TO-6350 & Burns 1991, 1996 & \\
\hline \multicolumn{6}{|c|}{ Bluefish Caves, Yukon Territory } \\
\hline \multirow[t]{7}{*}{ Cave I } & Moose (Alces alces) & $(11,570 \pm 60)$ & CAMS-23472 & $\begin{array}{l}\text { Pers. comm., J. } \\
\text { Cinq-Mars } 2010\end{array}$ & \\
\hline & $\begin{array}{l}\text { Caribou (Rangifer } \\
\text { tarandus) }\end{array}$ & $(12,210 \pm 210)$ & RIDDL-277 & $\begin{array}{l}\text { Pers. comm., J. } \\
\text { Cinq-Mars } 2010\end{array}$ & \\
\hline & $\begin{array}{l}\text { Caribou (Rangifer } \\
\text { tarandus) }\end{array}$ & $(12,830 \pm 60)$ & CAMS-23468 & $\begin{array}{l}\text { Pers. comm., J. } \\
\text { Cinq-Mars } 2010\end{array}$ & \\
\hline & $\begin{array}{l}\text { Mammoth } \\
\text { (Mammuthus sp.) }\end{array}$ & $(12,845 \pm 250)$ & CRNL-1220 & $\begin{array}{l}\text { Pers. comm., J. } \\
\text { Cinq-Mars } 2010\end{array}$ & $\begin{array}{l}\text { Butchered bone. } \\
\text { Normalized age is } \\
\text { average of three runs. }\end{array}$ \\
\hline & $\begin{array}{l}\text { Bighorn sheep (cf. } \\
\text { Ovis canadensis } \\
\text { probably } O \text {. dalli) }\end{array}$ & $(13,580 \pm 80)$ & CAMS-23473 & $\begin{array}{l}\text { Pers. comm., J. } \\
\text { Cinq-Mars } 2010\end{array}$ & \\
\hline & $\begin{array}{l}\text { Woolly mammoth } \\
\text { (Mammuthus } \\
\text { primigenius) }\end{array}$ & $(13,940 \pm 160)$ & RIDDL-559 & $\begin{array}{l}\text { Pers. comm., J. } \\
\text { Cinq-Mars } 2010\end{array}$ & \\
\hline & $\begin{array}{l}\text { Yukon horse } \\
\text { (Equus lambei) }\end{array}$ & $(17,440 \pm 220)$ & RIDDL-278 & $\begin{array}{l}\text { Pers. comm., J. } \\
\text { Cinq-Mars } 2010\end{array}$ & \\
\hline \multirow[t]{8}{*}{ Cave II } & Bison (cf. Bison sp.) & $(10,230 \pm 140)$ & RIDDL-561 & $\begin{array}{l}\text { Pers. comm., J. } \\
\text { Cinq-Mars } 2010\end{array}$ & \\
\hline & $\begin{array}{l}\text { Mammoth } \\
\text { (Mammuthus sp.) }\end{array}$ & $\begin{array}{c}15,500 \pm 130 \\
(15,540 \pm 130)\end{array}$ & GSC-3053 & $\begin{array}{l}\text { Pers. comm., J. } \\
\text { Cinq-Mars } 2010\end{array}$ & \\
\hline & $\begin{array}{l}\text { Mammoth } \\
\text { (Mammuthus sp.) }\end{array}$ & $(17,880 \pm 330)$ & CRNL-1221 & $\begin{array}{l}\text { Pers. comm., J. } \\
\text { Cinq-Mars } 2010\end{array}$ & Butchered bone. \\
\hline & $\begin{array}{l}\text { Mammoth } \\
\text { (Mammuthus sp.) }\end{array}$ & $(19,640 \pm 170)$ & RIDDL-330 & $\begin{array}{l}\text { Pers. comm., J. } \\
\text { Cinq-Mars } 2010\end{array}$ & \\
\hline & $\begin{array}{l}\text { Mammoth } \\
\text { (Mammuthus sp.) }\end{array}$ & $(20,230 \pm 180)$ & RIDDL-223 & $\begin{array}{l}\text { Pers. comm., J. } \\
\text { Cinq-Mars } 2010\end{array}$ & \\
\hline & $\begin{array}{l}\text { Mammoth } \\
\text { (Mammuthus sp.) }\end{array}$ & $(22,740 \pm 90)$ & CAMS-23470 & $\begin{array}{l}\text { Pers. comm., J. } \\
\text { Cinq-Mars } 2010\end{array}$ & \\
\hline & Horse (Equus sp.) & $\begin{array}{c}22,680 \pm 530 \\
(22,760 \pm 530)\end{array}$ & CRNL-1237 & $\begin{array}{l}\text { Pers. comm., J. } \\
\text { Cinq-Mars } 2010\end{array}$ & \\
\hline & $\begin{array}{l}\text { Mammoth } \\
\text { (Mammuthus sp.) }\end{array}$ & $(23,200 \pm 250)$ & RIDDL-225 & $\begin{array}{l}\text { Pers. comm., J. } \\
\text { Cinq-Mars } 2010\end{array}$ & $\begin{array}{l}\text { Butchered bone } \\
\text { core. }\end{array}$ \\
\hline
\end{tabular}


Table 2. Continued.

\begin{tabular}{|c|c|c|c|c|c|}
\hline Cave & Species & $\begin{array}{l}\text { Radiocarbon } \\
\text { Age }(\mathrm{BP})\end{array}$ & Lab Number ${ }^{\mathrm{a}}$ & Reference & Remarks \\
\hline & $\begin{array}{l}\text { Mammoth } \\
\text { (Mammuthus sp.) }\end{array}$ & $(23,910 \pm 200)$ & RIDDL-224 & $\begin{array}{l}\text { Pers. comm., J. } \\
\text { Cinq-Mars } 2010\end{array}$ & $\begin{array}{l}\text { Butchered bone. } \\
\text { Bone flake that } \\
\text { can be refitted to } \\
\text { the above core. }\end{array}$ \\
\hline & $\begin{array}{l}\text { Caribou (Rangifer } \\
\text { tarandus) }\end{array}$ & $(24,820 \pm 115)$ & RIDDL-226 & $\begin{array}{l}\text { Pers. comm., J. } \\
\text { Cinq-Mars } 2010\end{array}$ & Butchered bone. \\
\hline & Bison (Bison sp.) & $(31,730 \pm 230)$ & CAMS-23469 & $\begin{array}{l}\text { Pers. comm., J. } \\
\text { Cinq-Mars } 2010\end{array}$ & \\
\hline & $\begin{array}{l}\text { Snow Goose (Chen } \\
\text { caerulescens) }\end{array}$ & $\begin{array}{l}7670 \pm 60 \\
(7780 \pm 60)\end{array}$ & Beta-126870 & $\begin{array}{l}\text { Pers. comm., J. } \\
\text { Cinq-Mars } 2010\end{array}$ & $\begin{array}{l}\text { Bone has cutmarks } \\
\text { indicating human } \\
\text { butchering. }\end{array}$ \\
\hline & $\begin{array}{l}\text { Caribou or Dall sheep } \\
\text { (Rangifer tarandus } \\
\text { or Ovis dalli) }\end{array}$ & $\begin{array}{c}21,030 \pm 150 \\
(21,100 \pm 150)\end{array}$ & Beta-140679 & $\begin{array}{l}\text { Pers. comm., J. } \\
\text { Cinq-Mars } 2010\end{array}$ & \\
\hline \multirow[t]{9}{*}{ Cave III } & $\begin{array}{l}\text { Wapiti or elk (Cervus } \\
\text { elaphus) }\end{array}$ & $(10,820 \pm 60)$ & CAMS-23467 & $\begin{array}{l}\text { Pers. comm., J. } \\
\text { Cinq-Mars } 2010\end{array}$ & \\
\hline & Horse (Equus sp.) & $\begin{array}{c}12,290 \pm 440 \\
(12,370 \pm 440)\end{array}$ & CRNL-1236 & $\begin{array}{l}\text { Pers. comm., J. } \\
\text { Cinq-Mars } 2010\end{array}$ & Butchered bone. \\
\hline & $\begin{array}{l}\text { Snowy Owl (Nyctea } \\
\text { scandiaca) }\end{array}$ & $(13,350 \pm 100)$ & Beta-129151 & $\begin{array}{l}\text { Pers. comm., J. } \\
\text { Cinq-Mars } 2010\end{array}$ & \\
\hline & $\begin{array}{l}\text { Saiga antelope (Saiga } \\
\text { tatarica) }\end{array}$ & $(13,390 \pm 180)$ & RIDDL-279 & $\begin{array}{l}\text { Pers. comm., J. } \\
\text { Cinq-Mars } 2010\end{array}$ & \\
\hline & $\begin{array}{l}\text { Tundra muskox } \\
\text { (Ovibos moschatus) }\end{array}$ & $(14,370 \pm 130)$ & RIDDL-557 & $\begin{array}{l}\text { Pers. comm., J. } \\
\text { Cinq-Mars } 2010\end{array}$ & \\
\hline & $\begin{array}{l}\text { Cougar (Puma } \\
\text { concolor) }\end{array}$ & $(18,970 \pm 1490)$ & TO-1266 & $\begin{array}{l}\text { Pers. comm., J. } \\
\text { Cinq-Mars } 2010\end{array}$ & \\
\hline & $\begin{array}{l}\text { Mammoth } \\
\text { (Mammuthus sp.) }\end{array}$ & $(22,430 \pm 260)$ & RIDDL-558 & $\begin{array}{l}\text { Pers. comm., J. } \\
\text { Cinq-Mars } 2010\end{array}$ & \\
\hline & $\begin{array}{l}\text { Steppe bison (Bison } \\
\text { priscus) }\end{array}$ & $(23,710 \pm 100)$ & CAMS-23471 & $\begin{array}{l}\text { Pers. comm., J. } \\
\text { Cinq-Mars } 2010\end{array}$ & \\
\hline & $\begin{array}{l}\text { Beringian ferret } \\
\text { (Mustela } \\
\text { eversmanni) }\end{array}$ & $(33,550 \pm 350)$ & TO-1196 & $\begin{array}{l}\text { Pers. comm., J. } \\
\text { Cinq-Mars } 2010\end{array}$ & \\
\hline \multirow{9}{*}{$\begin{array}{l}\text { Charlie Lake } \\
\text { Cave, British } \\
\text { Columbia }\end{array}$} & $\begin{array}{r}\text { Common Raven } \\
\text { (Corvus corax) }\end{array}$ & $10,290 \pm 100$ & CAMS-2317 & Driver 1999 & \\
\hline & $\begin{array}{r}\text { Common Raven } \\
\text { (Corvus corax) }\end{array}$ & $9490 \pm 140$ & CAMS-2318 & Driver 1999 & \\
\hline & Bison (Bison sp.) & $10,770 \pm 120$ & SFU-454 & Harington $2003 \mathrm{~b}$ & \\
\hline & Bison (Bison sp.) & $(10,560 \pm 80)$ & CAMS-2134 & Harington $2003 \mathrm{~b}$ & \\
\hline & Bison (Bison sp.) & $10,450 \pm 150$ & SFU-300 & Harington $2003 \mathrm{~b}$ & \\
\hline & Bison (Bison sp.) & $10,380 \pm 160$ & SFU-378 & Harington 2003b & \\
\hline & Bison (Bison sp.) & $(9980 \pm 150)$ & RIDDL-393 & Harington 2003b & \\
\hline & Bison (Bison sp.) & $9760 \pm 160$ & SFU-355 & Harington $2003 \mathrm{~b}$ & \\
\hline & Bison (Bison sp.) & $(9670 \pm 150)$ & CAMS-2136 & Harington $2003 \mathrm{~b}$ & \\
\hline $\begin{array}{l}\text { Resonance } \\
\text { Cave, British } \\
\text { Columbia }\end{array}$ & $\begin{array}{l}\text { Mountain goat } \\
\text { (Oreamnos } \\
\text { americanus) }\end{array}$ & $(12,200 \pm 190)$ & TO-6072 & Nagorsen and Keddie 2000 & \\
\hline \multirow[t]{3}{*}{$\begin{array}{l}\text { Pellucidar II } \\
\text { Cave, British } \\
\text { Columbia }\end{array}$} & $\begin{array}{l}\text { Mountain goat } \\
\text { (Oreamnos } \\
\text { americanus })\end{array}$ & $(12,070 \pm 70)$ & TO-5006 & Nagorsen and Keddie 2000 & \\
\hline & Deer (Odocoileus sp.) & $8710 \pm 25$ & UCIAMS-41053 & Steffen et al. 2008 & \\
\hline & $\begin{array}{c}\text { Black bear (Ursus } \\
\text { americanus) }\end{array}$ & $11,110 \pm 30$ & UCIAMS-41052 & Steffen et al. 2008 & \\
\hline
\end{tabular}


Table 2. Continued.

\begin{tabular}{|c|c|c|c|c|c|}
\hline Cave & Species & $\begin{array}{l}\text { Radiocarbon } \\
\text { Age }(\mathrm{BP})\end{array}$ & Lab Number ${ }^{\mathrm{a}}$ & Reference & Remarks \\
\hline & $\begin{array}{c}\text { Mammal bone } \\
\text { (Mammalia) }\end{array}$ & $11,465 \pm 30$ & UCIAMS-41046 & Steffen et al. 2008 & \\
\hline & $\begin{array}{l}\text { Mammal bone } \\
\text { (Mammalia) }\end{array}$ & $11,580 \pm 30$ & UCIAMS-41045 & Steffen et al. 2008 & \\
\hline & $\begin{array}{l}\text { Giant short-faced bear } \\
\text { (Arctodus simus) }\end{array}$ & $11,615 \pm 30$ & UCIAMS-41049 & Steffen et al. 2008 & \\
\hline & $\begin{array}{l}\text { Mammal bone } \\
\text { (Mammalia) }\end{array}$ & $11,725 \pm 30$ & UCIAMS-41047 & Steffen et al. 2008 & \\
\hline & $\begin{array}{l}\text { Giant short-faced bear } \\
\text { (Arctodus simus) }\end{array}$ & $11,775 \pm 30$ & UCIAMS-41048 & Steffen et al. 2008 & \\
\hline & $\begin{array}{l}\text { Brown bear (Ursus } \\
\text { arctos) }\end{array}$ & $12,425 \pm 35$ & UCIAMS-41050 & Steffen et al. 2008 & \\
\hline & $\begin{array}{l}\text { Brown bear (Ursus } \\
\text { arctos) }\end{array}$ & $12,440 \pm 35$ & UCIAMS-41051 & Steffen et al. 2008 & \\
\hline \multirow[t]{8}{*}{$\begin{array}{l}\text { Port Eliza } \\
\text { Cave, British } \\
\text { Columbia }\end{array}$} & $\begin{array}{l}\text { Mountain goat } \\
\text { (Oreamnos } \\
\text { americanus) }\end{array}$ & $12,340 \pm 50$ & CAMS-97342 & Al-Suwaidi et al. 2006 & \\
\hline & $\begin{array}{l}\text { Mountain goat } \\
\text { (Oreamnos } \\
\text { americanus) }\end{array}$ & $16,340 \pm 60$ & CAMS-102798 & Al-Suwaidi et al. 2006 & \\
\hline & $\begin{array}{l}\text { Savannah Sparrow } \\
\quad \text { (Passerculus } \\
\text { sandwichensis) }\end{array}$ & $16,270 \pm 170$ & CAMS-88275 & Al-Suwaidi et al. 2006 & \\
\hline & Vole (Microtus sp.) & $18,010 \pm 100$ & CAMS-74624 & Al-Suwaidi et al. 2006 & \\
\hline & Vole (Microtus sp.) & $16,340 \pm 60$ & CAMS-74625 & Al-Suwaidi et al. 2006 & \\
\hline & Vole (Microtus sp.) & $17,100 \pm 70$ & CAMS-102797 & Al-Suwaidi et al. 2008 & \\
\hline & Marmot (Marmota sp.) & $16,460 \pm 170$ & CAMS-88274 & Al-Suwaidi et al. 2008 & $\begin{array}{l}\text { Duplicate analysis } \\
\text { of same bone } \\
\text { fragment. }\end{array}$ \\
\hline & Marmot (Marmota sp.) & $16,965 \pm 45$ & CAMS-97341 & Al-Suwaidi et al. 2008 & $\begin{array}{l}\text { Duplicate analysis } \\
\text { of same bone } \\
\text { fragment. }\end{array}$ \\
\hline $\begin{array}{l}\text { Windy Link } \\
\text { Pot Cave, } \\
\text { British Columbia }\end{array}$ & $\begin{array}{l}\text { Black bear (Ursus } \\
\text { americanus) }\end{array}$ & $\begin{array}{c}9760 \pm 140 \\
(9830 \pm 140)\end{array}$ & Beta-10714 & Nagorsen et al. 1995 & $\begin{array}{l}\text { Composite sample } \\
\text { of two tibias, } \\
\text { three ribs and two } \\
\text { vertebrae. }\end{array}$ \\
\hline \multirow[t]{6}{*}{$\begin{array}{l}\text { Mariner Mountain } \\
\text { Cave, British } \\
\text { Columbia }\end{array}$} & $\begin{array}{l}\text { Vancouver Island } \\
\text { marmot (Marmota } \\
\text { vancouverensis) }\end{array}$ & $890 \pm 50$ & TO-3562 & Nagorsen et al. 1996 & \\
\hline & $\begin{array}{l}\text { Vancouver Island } \\
\text { marmot (Marmota } \\
\text { vancouverensis) }\end{array}$ & $920 \pm 50$ & TO-3563 & Nagorsen et al. 1996 & \\
\hline & $\begin{array}{l}\text { Vancouver Island } \\
\text { marmot (Marmota } \\
\text { vancouverensis) }\end{array}$ & $990 \pm 50$ & TO-3564 & Nagorsen et al. 1996 & \\
\hline & $\begin{array}{l}\text { Vancouver Island } \\
\text { marmot (Marmota } \\
\text { vancouverensis) }\end{array}$ & $850 \pm 50$ & TO-3565 & Nagorsen et al. 1996 & \\
\hline & $\begin{array}{l}\text { Vancouver Island } \\
\text { marmot (Marmota } \\
\text { vancouverensis) }\end{array}$ & $890 \pm 50$ & TO-3566 & Nagorsen et al. 1996 & \\
\hline & $\begin{array}{l}\text { Vancouver Island } \\
\text { marmot (Marmota } \\
\text { vancouverensis) }\end{array}$ & $970 \pm 90$ & TO-3567 & Nagorsen et al. 1996 & \\
\hline
\end{tabular}


Table 2. Continued.

\begin{tabular}{|c|c|c|c|c|c|}
\hline Cave & Species & $\begin{array}{l}\text { Radiocarbon } \\
\text { Age(BP) }\end{array}$ & Lab Number ${ }^{\mathrm{a}}$ & Reference & Remarks \\
\hline $\begin{array}{l}\text { Golden Hinde } \\
\text { Cave, British } \\
\text { Columbia }\end{array}$ & $\begin{array}{l}\text { Vancouver Island } \\
\text { marmot (Marmota } \\
\text { vancouverensis) }\end{array}$ & $830 \pm 60$ & TO-4265 & Nagorsen et al. 1996 & \\
\hline \multirow[t]{2}{*}{$\begin{array}{l}\text { Clayoquot } \\
\text { Plateau Cave, } \\
\text { British Columbia }\end{array}$} & $\begin{array}{l}\text { Vancouver Island } \\
\text { marmot (Marmota } \\
\text { vancouverensis) }\end{array}$ & $2630 \pm 50$ & TO-1224 & Nagorsen et al. 1996 & \\
\hline & $\begin{array}{l}\text { Vancouver Island } \\
\text { marmot (Marmota } \\
\text { vancouverensis) }\end{array}$ & $2490 \pm 50$ & TO-692 & Nagorsen et al. 1996 & \\
\hline \multirow{2}{*}{$\begin{array}{l}\text { Limestone } \\
\text { Mountain } \\
\text { Cave, British } \\
\text { Columbia }\end{array}$} & $\begin{array}{l}\text { Vancouver Island } \\
\text { marmot (Marmota } \\
\text { vancouverensis) }\end{array}$ & $1030 \pm 60$ & TO-4266 & Nagorsen et al. 1996 & \\
\hline & $\begin{array}{l}\text { Vancouver Island } \\
\text { marmot (Marmota } \\
\text { vancouverensis) }\end{array}$ & $1070 \pm 60$ & ТO-4267 & Nagorsen et al. 1996 & \\
\hline \multicolumn{6}{|c|}{$\begin{array}{l}\text { "a Radiocarbon-Date Laboratories: Beta - Beta Analytic Inc., Miami, Florida; CAMS - Center for Accelerator Mass Spectrometry, Lawrence Livermore Laboratories, } \\
\text { California; CRNL - Chalk River Nuclear Laboratories, Chalk River, Ontario (no longer operating); GaK - Gakushuin University, Tokyo; GSC - Geological Survey of } \\
\text { Canada, Ottawa, Ontario; QU - Centre de Recherche Minérales, Ministère des Richesses Naturelles, Gouvernment du Québec (no longer operating); RIDDL - Radioisotope } \\
\text { Direct Detection Laboratory, McMaster University, Hamilton, Ontario (no longer operating); SFU - Simon Fraser University, Burnaby, British Columbia (no longer } \\
\text { operating); TO - Isotrace Laboratory, University of Toronto, Toronto, Ontario; UCIAMS - Keck Carbon Cycle AMS Facility, Earth System Science Department, University } \\
\text { of California, Irvine, California. }\end{array}$} \\
\hline
\end{tabular}

pedidae, a small rail of family Rallidae, a small wader of order Charadriiformes, the common raven Corvus corax, perching birds of order Passeriformes, and surface-feeding ducks of tribe Anatini, including the green-winged teal Anas crecca, the mallard Anas platyrhynchos, and the ruddy duck Oxyura jamaicensis. Two raven skeletons associated with Paleoindian occupations dated about 10,500 and 9500 B.P. were evidently deposited deliberately by people. Among the mammals, the snowshoe hare, the ground squirrel, small rodents including the collared lemming, and bison were most commonly represented. A small assemblage of bison Bison sp. from the Paleoindian components at the cave seem to have resulted from storage of frozen bison limbs in a series of meat caches that would have been difficult for scavengers to access. Other mammals were a large (possibly arctic) hare, a woodchuck or marmot Marmota sp., the deer mouse, the southern red-backed vole Myodes (Clethrionomys) gapperi, the meadow or long-tailed vole, the taiga vole Microtus xanthognathus, other unidentified rodents, the wolf or dog Canis lupus/familiaris, the least weasel Mustela nivalis, another small weasel, and a deer. These fossils indicate that from about 10,500 to 10,000 B.P. the landscape was open, with some water, marshes, and patches of forest, changing to forest about 10,000 years ago. By 9,000 years ago, the fauna was modern (Driver, 1988, 1998a, 1998b, 1999, 2001; Driver and Vallières, 2008; Driver et al., 1996; Harington, 1996).

Several caves on Vancouver Island (Fig. 1b), have yielded vertebrate remains. Since 1985, remains of the
Vancouver Island marmot Marmota vancouverensis have been discovered in four high-elevation cave sites: Clayoquot Plateau, Mariner Mountain, Limestone Mountain, and Golden Hinde. Artifacts and cut marks on bones recovered in Mariner Mountain Cave indicate that the marmot remains result from human hunting. Radiocarbon dates show that these sites are prehistoric, ranging from 2,630 to 830 B.P. The remains indicate a range decline in this marmot. Although the black bear, the Columbian black-tailed deer Odocoileus hemionus columbianus, the American marten, and the red squirrel are represented in the fauna, the predominance of Vancouver Island marmots suggests that aboriginal people traveled to these remote areas to hunt marmots (Nagorsen et al., 1996).

Although the mountain goat is now absent from most Pacific Coast islands, including Vancouver Island, 12,000year-old skeletal remains were found in Resonance and Pellucidar caves east of Nimpkish Lake on northern Vancouver Island. The caves are $5 \mathrm{~km}$ apart and are situated at an elevation of about $800 \mathrm{~m}$. Limb bones of these Pleistocene mountain goats are within the size range of the modern species, suggesting a postglacial origin. Mountain goats probably became extinct on Vancouver Island during the Early Holocene (Nagorsen and Keddie, 2000).

Pellucidar II Cave is situated near Pellucidar I and Resonance caves on the eastern slope of Nimpkish Lake, at an elevation of $480 \mathrm{~m}$. It is of interest because of the relatively large fauna represented there. The Late Pleistocene fauna consists of a hawk Buteo sp., a squirrel, a mouse

Journal of Cave and Karst Studies, December 2011 175 


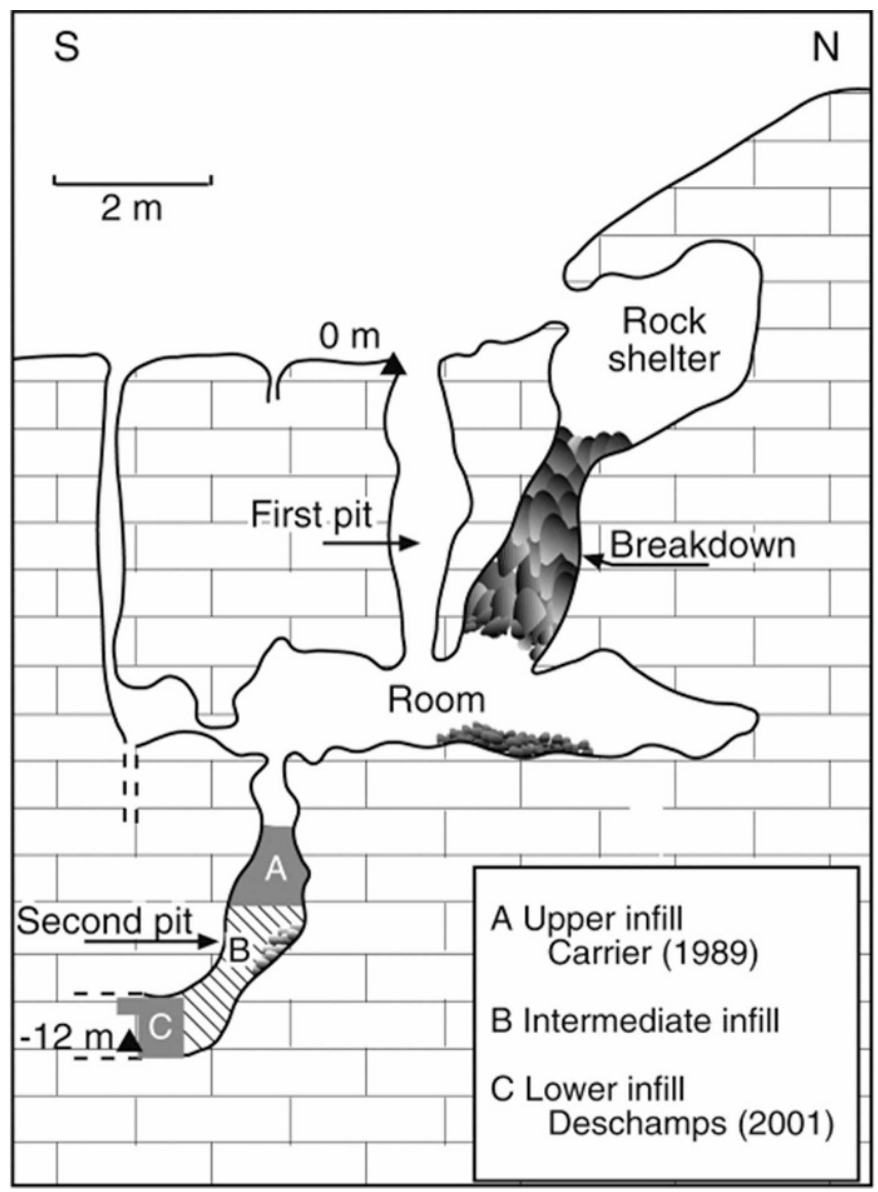

Figure 2. Mine Cave, Quebec. Cross-section diagram of the cave (Lauriol, et al., 2003).

Peromyscus sp., a shrew Sorex sp., a deer Odocoileus sp., the mountain goat, and several bears (extinct giant shortfaced bear Arctodus simus, black bear, and brown bear). AMS radiocarbon dates on these bones vary from about 12,500 B.P. for a brown bear humerus to about 11,000 B.P. for a black bear humerus. Palatine and humerus fragments from the giant short-faced bear yielded radiocarbon ages of about 11,600 and 11,800 B.P., respectively. Specimens inferred to be from the Holocene on the basis of stratigraphic context include the blue grouse Dendragrapus obscurus, the white-winged crossbill Loxia leucoptera, a toad Bufo sp., a small snake, the red squirrel, western heather vole Phenacomys intermedius, another vole Microtus sp., a mouse Peromyscus sp., the marmot cf. Marmota vancouverensis, a small bat Myotis sp., the black bear, and a deer Odocoileus sp. The deer humerus was radiocarbon dated to about 8700 B.P. Preliminary results (Steffen et al., 2008) show that by 12,000 B.P. the coastal environment of northern Vancouver Island sustained three species of bear and other vertebrates that could have provided abundant resources for people.

Port Eliza Cave, a raised sea cave, is located on the northwestern coast of Vancouver Island at an elevation of

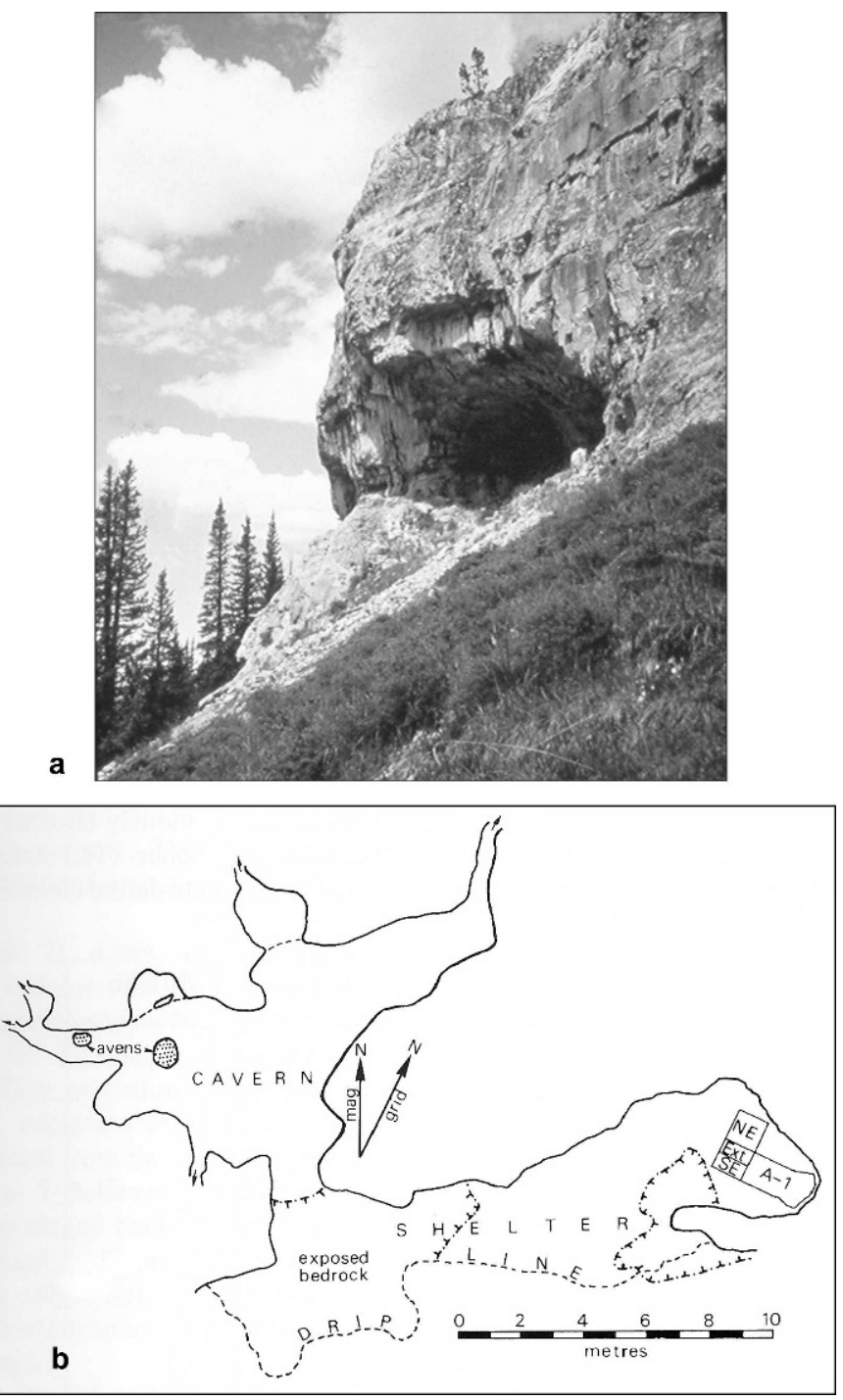

Figure 3. January Cave, Alberta. (a) Photograph of the cave entry. (b) Plan view; four test pits are marked at the far right (Burns, 1991).

$85 \mathrm{~m}$, well above the $40 \mathrm{~m}$ postglacial sea level maximum (Fig. 6a, b). It formed along a fault trace and is $60 \mathrm{~m}$ long, varying in height from about 1 to $15 \mathrm{~m}$. A diverse fish, amphibian, bird, and mammal fauna (about 3,600 specimens) was recorded from a basal unit of silty-sandy sediment greater than $50 \mathrm{~cm}$ deep. The fauna, yielding ages of 18,000 to 16,000 B.P., includes a salmon Onchorhynchus sp., the cutthroat trout Onchorhynchus clarkii, the threespined stickleback Gasterostens aculeatus, a greenling of family Hexagrammidae, the Alaska pollock Theragra chalcogramma, a flatfish of order Pleuronectiformes, an Irish lord Hemilepidotus sp., a sculpin of family Cottidae, the Pacific tomcod Microgadus proximus, the western toad Bufo boreas, the red-throated loon Gavia stellata, a small alcid of family Alcidae, a cormorant Phalacrocorax sp., a duck, the horned lark Eromophila alpestris, the Savanna 

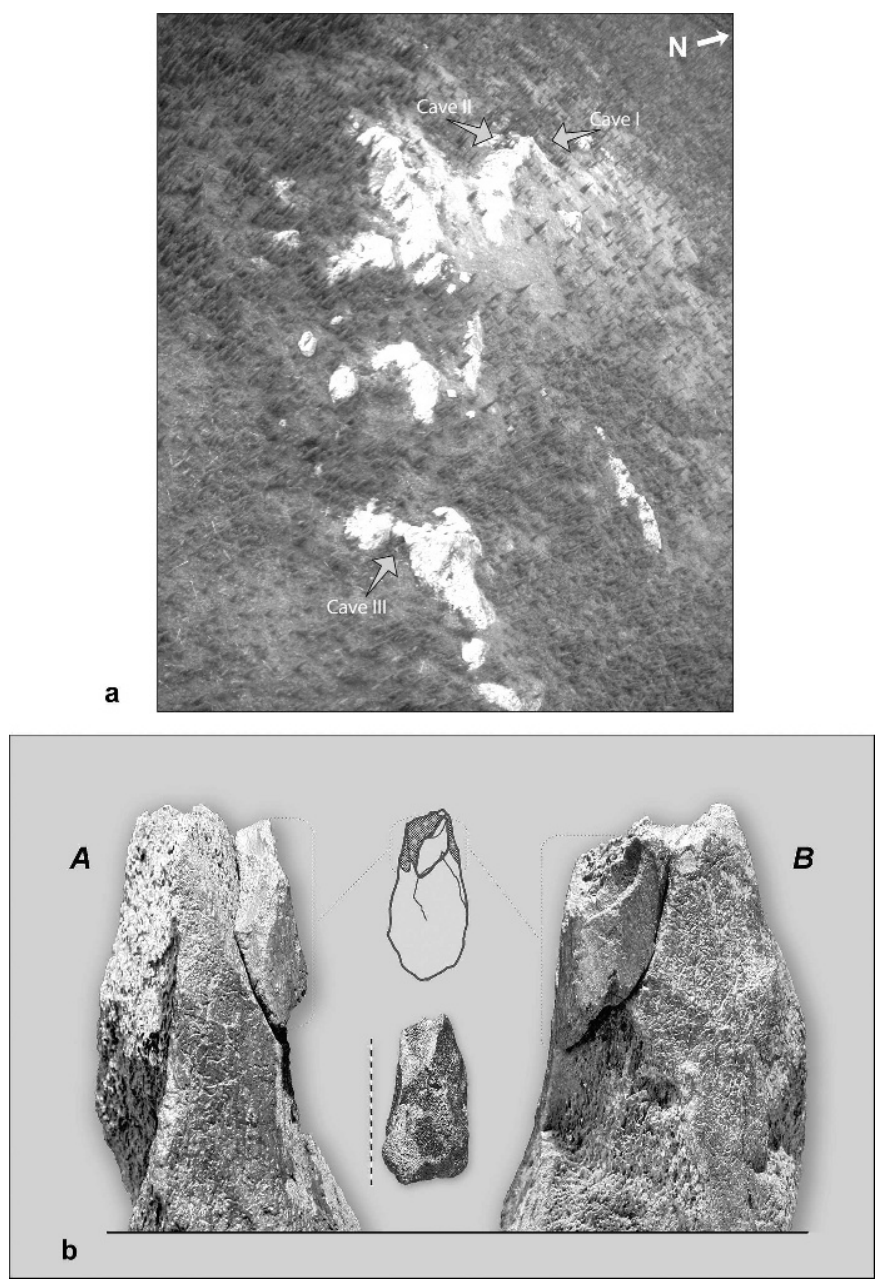

Figure 4. Bluefish Caves, Yukon. (a) Aerial view of Bluefish Caves (I-III) situated near the base of a Devonian limestone ridge. (b) A flaked mammoth bone core and its refitted flake from the lower loess unit at Bluefish Cave II, dated to around 24,000 B.P. A and B, detailed side views of the core and refitted flake; top center, schematic sketch of frontal view of core showing the position of three flake scars and the refitted flake; bottom center, frontal view of the bone core with $25 \mathrm{~cm}$ scale (Harington and Cinq-Mars, 2008).

sparrow Passerculus sandwichensis, the Townsend's vole Microtus townsendii, the long-tailed vole, the heather vole Phenacomys intermedius, the hoary marmot, the American marten, and the noble marten Martes americana nobilis, as well as the mountain goat and a canid (evidence from tooth marks). Fishes suggest that the shore was close enough for predators to have introduced this material to the cave. The terrestrial fauna indicates a cool, open environment with maximum summer temperatures cooler than present. A postglacial fauna consists of a mouse Peromyscus sp. and the mountain goat. Humans could have survived here on a mixed marine-terrestrial diet, confirming the viability of the Pacific coastal migration hypothesis for this part of the proposed route (Ward et al., 2003; Al-Suwaidi et al., 2006).

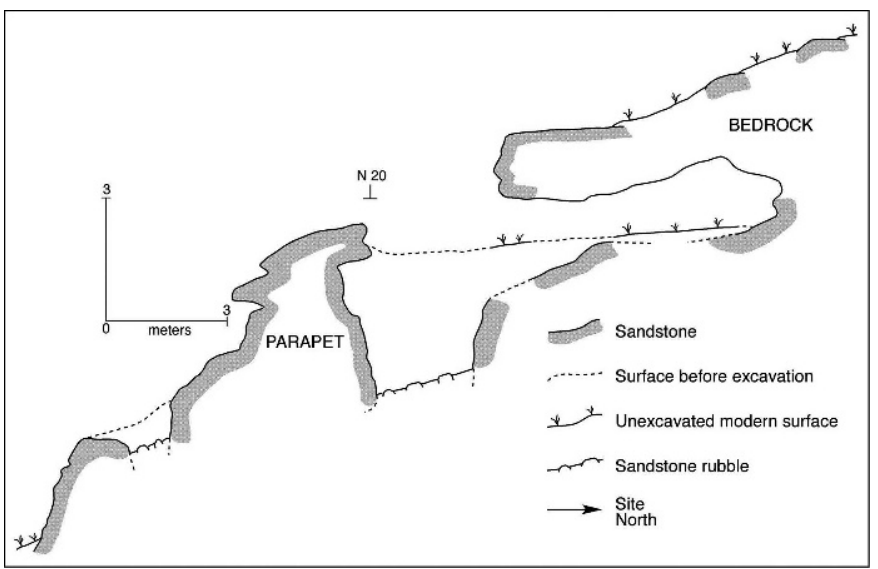

Figure 5. Charlie Lake Cave, British Columbia. Crosssection of the cave (Driver and Vallières, 2008).
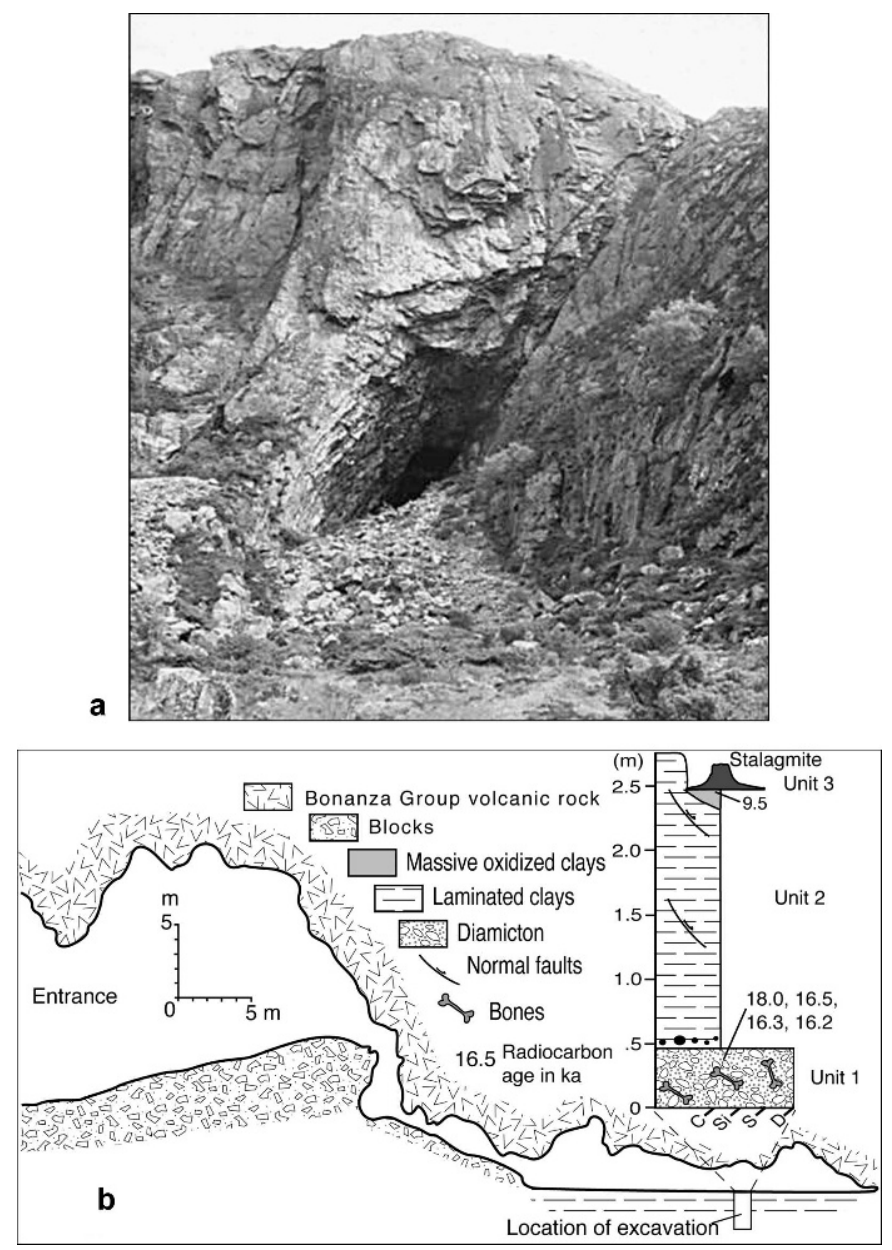

Figure 6. Port Eliza Cave, British Columbia. (a) Photograph of the cave entry. (b) Cross-section diagram of the cave (Al-Suwaidi, et al., 2006).

Journal of Cave and Karst Studies, December 2011 177 
In 1983, cavers discovered skeletal remains of three Early Holocene $(9,830 \pm 140$ B.P.) black bears at Windy Link Pot Cave $\left(49^{\circ} 46^{\prime} 55^{\prime \prime} \mathrm{N}, 125^{\circ} 59^{\prime} 10^{\prime \prime} \mathrm{W}\right)$, situated at an elevation of about $900 \mathrm{~m}$ near Gold River on west-central Vancouver Island. The bears were relatively large. This cave system extends for nearly $10 \mathrm{~km}$ underground, with several steep drops in the cave floor, so there are many natural traps. Bones of three black-tailed deer Odocoileus hemionus columbianus were also discovered nearby. The bones showed no evidence of butchery or carnivore tooth marks, so the animals presumably fell to their deaths. Pollen analysis of sediment adhering to the bones indicates that the bears lived in a mixed coniferous forest with a warmer, drier climate than today (Nagorsen et al., 1995; Harington, 1996).

\section{Conclusions}

This review of twenty-two ice age vertebrate faunas, with associated data, from Canadian caves is presented to make North American and other vertebrate paleontologists more aware of the Canadian evidence. It is hoped that this summary will stimulate further studies on the cave faunas mentioned, as well as the search for more Canadian caves containing fossils that add to our knowledge of the distribution of vertebrate species, their chronological sequence and paleoenvironments, such as four caves near the mouth of the Saguenay River reported by Brassard (Beaupré and Caron 1986, p. 240-241; Harington 2003b, p. 89) and Extinction Cave in southern Manitoba's Interlake region (Oosterom, 2009).

Finds in western Canadian caves are relevant to the question of what routes early people chose to enter North America. Two modified bone specimens from Bluefish Caves, Yukon, along with a series of AMS-dated bone cores and flakes from Old Crow Basin and a bison radioulna from Nugget Gulch near Dawson City, Yukon, imply that an early phase of human tool-making, often involving mammoth bone, began in Eastern Beringia as early as 40,000 years ago and ended about 25,000 years ago (CinqMars and Morlan, 1999; Harington and Morlan, 2002; Harington and Cinq-Mars, 2008). Further, Holen's (2006) evidence for human-modified mammoth skeletons from Kansas and Nebraska in the western United States suggests an entry-route to the heartland of North America east of the Rocky Mountains (the "ice-free corridor") before 18,000 years ago. Brown bear, with woolly mammoths (Lister and Bahn, 2007) and steppe bison (McDonald, 1981; Shapiro et al., 2004), likely reached the southern refugium in the unglaciated United States during the midWisconsin ice-free period about 26,000 B.P. (Matheus et al., 2004), so that route may have been feasible for humans too. And the Port Eliza Cave fauna, Vancouver Island, indicates that people could have survived there on a mixed marine-terrestrial diet, confirming the viability of a Pacific coastal migration as early as 18,000 to 16,000 B.P.
Preliminary results of the excavations at Pellucidar II Cave, Vancouver Island, show that by 12,000 B.P. the coastal vertebrate fauna could have provided abundant resources for human survival. So a later Pacific coastal migration could have occurred in latest Pleistocene-earliest Holocene time, as suggested by the oldest reliably dated, at nearly 10,000 B.P., human remains in Alaska discovered to the north in On Your Knees Cave, Prince of Wales Island (Dixon et al., 1997). Also, excavations on Haida Gwaii, Queen Charlotte Islands, since about 1995 have significantly enhanced our understanding of human history there. The archaeological record now extends to at least 10,500 B.P. (Fedje et al., 2004; Fedje and Mathewes, 2005).

Finally, it seems reasonable to suggest, as a follow-up to Ice Age Cave Faunas of North America (Schubert et al., 2003), that another book be published summarizing significant aspects of all North American cave faunas, perhaps following the pithy style of West's (1996) tome on the prehistory of Beringia.

\section{AcKnowledgements}

I thank Camille Ek for encouraging me to collect and study vertebrate remains from the caves near La Rédemption, Quebec; Bernard Lauriol and Luc Carrier (then one of his graduate students) for allowing me to accompany them to Mine Cave, Quebec, and to identify bones (many raccoon!) from the upper $100 \mathrm{~cm}$ excavation; Jacques Cinq-Mars for showing me Bluefish Caves, Yukon, and for facilitating study of their well-preserved large vertebrate remains as a research associate of the Canadian Museum of Civilization; Darlene McCuaig-Balkwill for providing me with additional information on bird remains that she identified from Bluefish Caves, Yukon; Martina Steffen for allowing me to study bear remains from Pellucidar II Cave, British Columbia; Bernard Lauriol, Jacques Cinq-Mars, Jim Burns, Jon Driver and Brent Ward for assistance with Figures 2-6. Also, Jim kindly provided a faunal list for Eagle Cave from his dissertation, and Jacques sent me a list of all radiocarbon dates for Bluefish Caves. Above all, I am grateful to Gail Harington for word processing and help with the tables and illustrations. The paper has greatly benefitted from comments by Chris Jass and an anonymous reviewer.

\section{REFERENCES}

Al-Suwaidi, M., Ward, B.C., Wilson, M.C., Hebda, R.J., Nagorsen, D.W., Marshall, D., Ghaleb, B., Wigen, R.J., and Enkin, R.J., 2006, Late Wisconsinan Port Eliza Cave deposits and their implications for human coastal migration: Geoarchaeology: An International Journal, v. 21 , no. 4 , p. $307-332$. doi: $10.1002 /$ gea. 20106 .

Bateman, R.M., 1961, Mammal occurrences in escarpment caves: Ontario Field Biologist, v. 15, p. 16-18.

Beaupré, M., and Caron, D., 1986. Découvrez le Québec souterrain. Sillery, Québec Science Editeur, and Sainte-Foy, Presses de l'Université du Québec. 254 p.

Beebe, B.F., 1983, Evidence of carnivore activity in a Late Pleistocene/ Early Holocene archaeological site (Bluefish Cave I), Yukon Territory, Canada, in Lemoine, G.M., and MacEachern, A.S., eds., 
Carnivores, Human Scavengers, and Predators: A Question of Bone Technology, Proceedings of the 15th Annual Conference of the Archaeological Association of the University of Calgary, University of Calgary, p. 1-14.

Burns, J.A., 1982, Water vole Microtus richardsoni (Mammalia, Rodentia) from the Late Pleistocene of Alberta: Canadian Journal of Earth Sciences, v. 19, p. 628-631.

Burns, J.A., 1987, Late Quaternary zoogeography of the northern pocket gopher, Thomomys talpoides, in southwestern Alberta: Canadian Field-Naturalist, v. 101, p. 419-422.

Burns, J.A., 1989, Fossil vertebrates from Rats Nest Cave, Alberta: Canadian Caver, v. 21, no. 1, p. 41-43.

Burns, J.A., 1991, Mid-Wisconsinan vertebrates and their environment from January Cave, Alberta, Canada: Quaternary Research, v. 35, p. 130-143. doi:10.1016/0033-5894(91)90100-J.

Burns, J.A., 2002, January Cave: an ancient window on the past: Alberta Archaeological Review, v. 37, p. 15-16.

Carrier, L., 1989, Le karst de Kingsmere: étude de ses remplissages, Parc de la Gatineau, Québec, [M.A. thesis], Université d'Ottawa, 120 p.

Churcher, C.S., and Fenton, M.B., 1968, Vertebrate remains from the Dickson Limestone Quarry, Halton County, Ontario, Canada: Bulletin of the National Speleological Society, v. 30, p. 11-16.

Churcher, C.S., and Karrow, P.F., 2008, The Hamilton Bar fauna: evidence for a Hypsithermal age. Canadian Journal of Earth Sciences, v. 45 , p. $1487-1500$.

Cinq-Mars, J., 1979, Bluefish Cave 1: a Late Pleistocene Eastern Beringian cave deposit in the northern Yukon: Canadian Journal of Archaeology, v. 3, p. 1-32.

Cinq-Mars, J., and Morlan, R.E., 1999, Bluefish Caves and Old Crow Basin: a new rapport, in Bonnichsen, R., and Turnmire, K.L., eds., Ice Age People of North America: Environment, Origins and Adaptations of the First Americans, Corvallis, Oregon State University Press for the Center for the Study of the First Americans, p. 200-212.

Dixon, E.J., Heaton, T.H., Fifield, T.E., Hamilton, T.D., Putnam, D.E., and Grady, F., 1997, Late Quaternary regional geoarchaeology of southeast Alaska karst: a progress report: Geoarchaeology: An International Journal, v. 12, no. 6, p. 689-712.

Driver, J.C., 1988, Late Pleistocene and Holocene vertebrates and palaeoenvironments from Charlie Lake Cave, northeast British Columbia: Canadian Journal of Earth Sciences, v. 25, p. 1545-1553.

Driver, J.C., 1998a. Human exploitation of pioneering post-glacial communities in northeastern British Columbia, Canada. 8th International Congress of the International Council for Archaeozoology (August 23-29, Victoria, British Columbia). Final Program and Abstracts, $107 \mathrm{p}$.

Driver, J.C., 1998b, Late Pleistocene collared lemming (Dicrostonyx torquatus) from northeastern British Columbia, Canada: Journal of Vertebrate Paleontology, v. 18, p. 816-818.

Driver, J.C., 1999, Raven skeletons from Paleoindian contexts, Charlie Lake Cave, British Columbia: American Antiquity, v. 64, p. 289-298.

Driver, J.C., 2001, Paleoecological and archaeological implications of the Charlie Lake Cave fauna, British Columbia 10,500 to 9500 B.P., in Gerlach, S.C., and Murray, M.S., eds., People and Wildlife in Northern North America: Essays in Honor of R. Dale Guthrie, Oxford, British Archaeological Reports International Series 944, p. $13-22$

Driver, J.C., and Vallières, C., 2008, The Paleoindian bison assemblage from Charlie Lake Cave, British Columbia: Canadian Journal of Archaeology, v. 32, p. 239-257.

Driver, J.C., Handly, M., Fladmark, K.R., Nelson, D.E., Sullivan, G.M., and Preston, R., 1996, Stratigraphy, radiocarbon dating, and culture history of Charlie Lake Cave, British Columbia: Arctic, v. 49, p. $265-277$

Fedje, D.W., and Mathewes, R.W., 2005, Conclusion: synthesis of environmental and archaeological data, in Fedje, D.W., and Mathewes, R.W., eds., Haida Gwaii: Human History and Environment from the Time of Loon to the Time of the Iron People, Vancouver, UBC Press, p. 372-375.

Fedje, D.W., Wigen, R.J., McLaren, D., and Mackie, Q., 2004, Archaeology and environment of karst landscapes in southern Haida Gwaii (Queen Charlotte Islands), West Coast, Canada: Paper presented at the 2004 Northwest Anthropological Conference, Eugene, Oregon. March.
Harington, C.R., 1980, A preliminary list of faunal remains from two caves (Trou Otis and Spéos de la Fée) in Gaspé, Québec, in Schroeder, J., ed., Le karst de plate-forme de Boischâtel et le karst barré de La Rédemption, état des connaissances, Montréal, Société Québecoise de Spéléologie, p. 95-105.

Harington, C.R., 1996, Quaternary animals: vertebrates of the ice age, in Ludvigsen, R., ed., Life in Stone: A Natural History of British Columbia's Fossils, Vancouver, UBC Press, p. 259-273.

Harington, C.R., 2003a, Quaternary vertebrates of Québec: a summary: Géographie physique et Quaternaire, v. 57, p. 85-94.

Harington, C.R., ed., 2003b, Annotated Bibliography of Quaternary Vertebrates of Northern North America - with Radiocarbon Dates, University of Toronto Press, $369 \mathrm{p}$.

Harington, C.R., 2006, Review of Blaine W. Schubert's, Jim I. Mead's, and Russell W. Graham's, eds., Ice Age Faunas of North America, The Palaeontology Newsletter, no. 61, p. 90-99.

Harington, C.R., and Cinq-Mars, J., 2008, Bluefish Caves - fauna and context, Beringian Research Notes, no. 19, p. 1-8.

Harington, C.R., and Morlan, R.E., 2002, Evidence for human modification of a Late Pleistocene bison (Bison sp.) bone from the Klondike District, Yukon Territory, Canada: Arctic, v. 55, no. 2, p. 143-147.

Holen, S.R., 2006, Taphonomy of two last glacial maximum mammoth sites in the central Great Plains of North America: a preliminary report on La Sena and Lovewell: Quaternary International, v. 142143(2006), p. 30-43.

Karrow, P.F., 2005, Quaternary geology of the Brampton Area, Ontario Geological Survey Report 257, 59 p.

Lasalle, P., 1984, Geological setting and preliminary faunal report for StElzéar Cave Québec, in Genoways, H.H., and Dawson, M.R., eds., Contributions in Quaternary Vertebrate Paleontology: A Volume in Memorial to John E. Guilday, Carnegie Museum of Natural History Special Publication, no. 8, p. 332-346.

Lasalle, P., and Guilday, J.E., 1980, Caverne de Saint-Elzéar-de-Bonaventure, Rapport preliminaire sur les fueilles de 1977 et 1978, Quebec, Édition du Ministère de l'Énergie et des Ressources, Direction générale de la recherche géologique et minérale, DPV-750, $31 \mathrm{p}$.

Lauriol, B., Deschamps, E., Carrier, L., Grimm, W., Morlan, R., and Talon, B., 2003, Cave infill and associated biotic remains as indicators of Holocene environment in Gatineau Park (Québec, Canada): Canadian Journal of Earth Sciences, v. 40, p. 789-803.

Lister, A., and Bahn, P., 2007, Mammoths: Giants of the Ice Age, Berkeley, University of California Press, $192 \mathrm{p}$

Matheus, P., Burns, J., Weinstock, J., and Hofreiter, M., 2004, Pleistocene brown bears in the mid-continent of North America: Science, v. 306, 1150 p. doi: 10.1126/science. 1101495 .

McCuaig-Balkwill, D., and Cinq-Mars, J., 1998, Migratory birds from Bluefish Caves, Eastern Beringia: $8^{\text {th }}$ International Congress of the International Council for Archaeozoology (August 23-29, Victoria, B.C.) Final Program and Abstracts, 194 p.

McDonald, J.N., 1981, North American Bison: Their Classification and Evolution, Berkeley, University of California Press, 316 p.

Mead, J.I., and Grady, F., 1996, Ochotona (Lagomorpha) from Late Quaternary cave deposits in eastern North America: Quaternary Research, v. 45, p. 93-101.

Morlan, R.E., 1989, Paleoecological implications of Late Pleistocene and Holocene microtine rodents from Bluefish Caves, northern Yukon Territory: Canadian Journal of Earth Sciences, v. 26, p. 149-156.

Nagorsen, D.W., and Keddie, G., 2000, Late Pleistocene mountain goats (Oreamnos americanus) from Vancouver Island: biogeographic implications: Journal of Mammalogy, v. 81, p. 666-675. doi: 10.1644/15451542(2000)081<0666:LPMGOA > 2.3.CO;2.

Nagorsen, D.W., Keddie, G., and Hebda, R.J., 1995, Early Holocene black bears, Ursus americanus, from Vancouver Island: Canadian Field-Naturalist, v. 109, p. 11-18

Nagorsen, D.W., Keddie, G., and Luszcz, T., 1996, Vancouver Island Marmot Bones from Subalpine Caves: Archaeological and Biological Significance, Victoria, British Columbia Parks, Occasional Paper No. $4,56 \mathrm{p}$.

Oosterom, N., 2009, Extinction Cave: unique hole in ground may yield bones of ice age creatures: The Beaver, v. 89, no. 2, 9 p.

Pigott, P., 1999, Tracking a phantom grizzly: Equinox, no. 102, p. 64-65, $68-74$.

Journal of Cave and Karst Studies, December 2011•179 
Savage, H., 1994, Prehistoric fauna in a vertical fissure cave in the Niagara Escarpment, Dufferin County, Ontario, in MacDonald, R.I., ed., Great Lakes Archaeology and Paleoecology: Exploring Interdisciplinary Initiatives for the Nineties: Proceedings of a Symposium presented by the Quaternary Sciences Institute, University of Waterloo, Waterloo, Ontario, September 21-22, 1991, Waterloo Ontario, Quaternary Sciences Institute publication 10.

Schubert, B.W., Mead, J.I., and Graham, R.W., eds., 2003, Ice Age Cave Faunas of North America, Bloomington, Indiana University Press, and Denver, Denver Museum of Nature and Science, 320 p.

Shapiro, B., and 27 coauthors, 2004, Rise and fall of the Beringian steppe bison: Science, v. 306, p. 1561-1565.
Steffen, M.L., Hebda, R.J., McLaren, D.S., and Fedje, D.W., 2008. P2 Cave: paleontological recovery and archaeological potential. Abstract of poster paper presented at the Northwest Anthropological Conference (April 23-26, 2008, Victoria, B.C.), Preliminary Schedule, 24 p.

Ward, B.C., Wilson, M.C., Nagorsen, D.W., Nelson, D.E., Driver, J.C., and Wigen, R.J., 2003, Port Eliza Cave: North American West Coast interstadial environment and implications for human migrations: Quaternary Science Reviews, v. 22, p. 1383-1388. doi:10.1016/S02773791(03)00092-1.

West, F.H., ed., 1996, American Beginnings: The Prehistory and Paleoecology of Beringia, Chicago, University of Chicago Press, $576 \mathrm{p}$. 